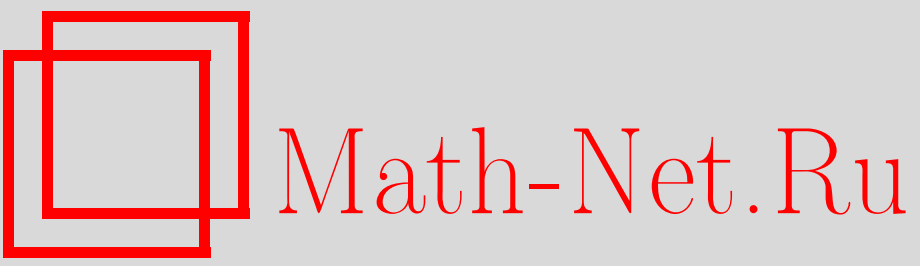

В. А. Ильин, Е. И. Моисеев, Оптимизация граничных управлений колебаниями струны, УМH, 2005, том 60, выпуск 6, 89-114

DOI: https://doi.org/10.4213/rm1678

Использование Общероссийского математического портала Math-Net.Ru подразумевает, что вы прочитали и согласны с пользовательским соглашением

http://www.mathnet.ru/rus/agreement

Параметры загрузки:

IP : 54.80 .73 .141

26 апреля 2023 г., 05:50:50 


\title{
ОПТИМИЗАЦИЯ ГРАНИЧНЫХ УПРАВЛЕНИЙ КОЛЕБАНИЯМИ СТРУНЫ
}

\author{
В. А. ИльИН, Е. И. МОИСЕЕВ
}

\begin{abstract}
В работе для болшого промежутка времени $T$ проводится оптимизация граничных управлений колебаниями струны для следующих семи задач: задачи граничного управления смещением на одном конце при условии, что второй конец или закреплен, или свободен, задачи граничного управления смещениями на двух концах, задачи граничного управления упругой силой на одном конце при условии, что второй конец или закреплен, или свободен, задачи граничного управления упругой силой на двух концах и задачи комбинированного граничного управления смещением на одном конце и упругой силой на другом конце. Оптимальные граничные управления в каждой из указанных семи задач ищутся как функции, доставляющие минимум соответствующему интегралу граничной энергии при наличии условий связи, вытекающих из выполнения в начальный момент времени $t=0$ заданных начальных условий и в финальный момент времени $t=T$ заданных финальных условий. Для всех семи задач оптимальные граничные управления предъявляются в явном аналитическом виде.

Библиография: 17 названий.
\end{abstract}

\section{СОДЕРЖАНИЕ}

Введение

$1^{\circ}$. Задача 1 (об оптимизации граничного управления смещением на одном конце при закрепленном втором конще)

$2^{\circ}$. Задача 2 (оптимизация граничного управления смещением на двух концах )

$3^{\circ}$. Задача 3 (оптимизация граничного управления смещением на одном конце при свободном втором конце)

$4^{\circ}$. Задача 4 (оптимизация граничного управления упругой силой на одном конце при закрепленном другом конце)

$5^{\circ}$. Задача 5 (оптимизация граничного управления упругой силой на одном конце при свободном втором конце)

$6^{\circ}$. Задача 6 (оптимизация граничных управлений упругой силой на двух концах

$7^{\circ}$. Задача 7 (оптимизация комбинированного граничного управления упругой силой на одном конце и смешением на другом конще) . ...

$8^{\circ}$. Обоснование основных утверждений, используемых при проведении

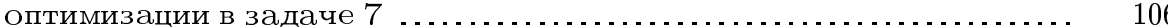

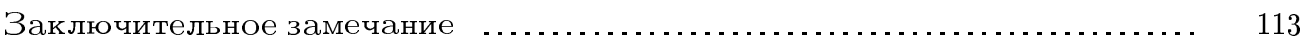

Список литературы ................................................ 113

(C) В. А. Ильин, Е. И. Моисеев 


\section{Введение}

В настоящей работе для большого промежутка времени $T$ в терминах обобщенного решения волнового уравнения $u_{t t}(x, t)-u_{x x}(x, t)=0$, допускаюшего сушествование в любой момент времени $t$ конечной энергии, проводится оптимизация семи задач граничного управления (граничного управления смешением на одном конце при закрепленном втором конще, граничного управления смещением на двух конщах, граничного управления смещением на одном конще при свободном втором конще, граничного управления упругой силой на одном конце при закрепленном втором конще, граничного управления упругой силой на одном конще при свободном втором конще, граничного управления упругой силой на двух конщах и комбинированного граничного управления упругой силой на одном конще и смешением на втором конце).

Во всех семи задачах в “длинном" прямоугольнике $Q_{T}=[0 \leqslant x \leqslant l] \times[0 \leqslant t \leqslant T]$ рассматривается допускающее существование конечной энергии обобшенное решение смешанной задачи для волнового уравнения

$$
u_{t t}(x, t)-u_{x x}(x, t)=0
$$

с произвольно заданными начальными условиями

$$
\left\{u(x, 0)=\varphi(x), u_{t}(x, 0)=\psi(x)\right\}
$$

и с такими граничными условиями первого или второго рода (частично, может быть, однородными), которые обеспечивают вьполнение в финальньй момент времени $t=T$ произвольно заданных финальных условий

$$
\left\{u(x, T)=\widehat{\varphi}(x), u_{t}(x, T)=\widehat{\psi}(x)\right\} .
$$

Это решение определяется как функция $u(x, t)$, удовлетворяющая тому интегральному тождеству, которое в случае гладкой функции $u(x, t)$ получается при перебрасывании в тождестве

$$
\int_{0}^{l} \int_{0}^{T}\left[u_{t t}(x, t)-u_{x x}(x, t)\right] \Phi(x, t) d x d t=0
$$

посредством интегрирования по частям производных функции $u(x, t)$ на соответствующие производные гладкой пробной функции $\Phi(x, t)$.

Однако проведенньй анализ показал, что указанное обобщенное решение $u(x, t)$ на самом деле является более гладким, чем традиционное обобщенное решение из класса $W_{2}^{1}\left(Q_{T}\right)$, и принадлежит впервые введенному В. А. Ильиным в работах [1] и [2] классу $\widehat{W}_{2}^{1}\left(Q_{T}\right)$, которьй определяется как множество функций двух переменных $u(x, t)$, непрерьвных в замкнутом прямоугольнике $\bar{Q}_{T}$ и имеюших в нем обе обобщенные частные производные $u_{x}(x, t)$ и $u_{t}(x, t)$, каждая из которых принадлежит не только классу $L_{2}\left(Q_{T}\right)$, но и классу $L_{2}[0 \leqslant x \leqslant l]$ при каждом $t$ из сегмента $[0, T]$ и классу $L_{2}[0 \leqslant t \leqslant T]$ при каждом $x$ из сегмента $[0, l]$.

В классе $\widehat{W}_{2}^{1}\left(Q_{T}\right)$ вся теория приобретает законченный характер, ибо необходимым условием принадлежности решения $u(x, t)$ этому классу является принадлежность начальных условий (2) указанными ниже классам:

$$
u(x, 0)=\varphi(x) \in W_{2}^{1}[0, l], \quad u_{t}(x, 0)=\psi(x) \in L_{2}[0, l],
$$

Работа выполнена при поддержке РФФИ (грант № 02-01-00324) и НШ-2042.2003.1. 
финальных функций (3) классам

$$
u(x, T)=\widehat{\varphi}(x) \in W_{2}^{1}[0, l], \quad u_{t}(x, T)=\widehat{\psi}(x) \in L_{2}[0, l],
$$

граничных условий, задаваемых смещениями, классу

$$
u(0, t)=\mu(t) \in W_{2}^{1}[0, T], \quad u(l, t)=\nu(t) \in W_{2}^{1}[0, T]
$$

и граничных условий, задаваемых упругими силами, классу

$$
u_{x}(0, t)=\mu(t) \in L_{2}[0, T], \quad u_{x}(l, t)=\nu(t) \in L_{2}[0, T] .
$$

При $T>l$ в случае граничных управлений на двух концах и при $T>2 l$ в случае граничного управления на одном конце сушествует бесконечно много различных граничных управлений, обеспечивающих переход колебательного процесса от произвольно заданных начальных условий (2) к произвольно заданным финальным условиям (3).

Поэтому при больших $T$ мы находим и предъявляем в явном аналитическом виде оптимальные граничные управления, которые доставляют минимум соответствуюшему интегралу граничной энергии при наличии условий связи, извлекаемых из вьполнения заданных начальных условий (2) и заданных финальных условий (3).

При этом минимизируемьй интеграл граничной энергии имеет следуюший вид:

$$
\int_{0}^{T}\left[\mu^{\prime}(t)\right]^{2} d t
$$

в случае граничного управления смещением $u(0, t)=\mu(t)$ на одном конце $x=0$;

$$
\int_{0}^{T}\left\{\left[\mu^{\prime}(t)\right]^{2}+\left[\nu^{\prime}(t)\right]^{2}\right\} d t
$$

в случае граничных управлений смешениями $u(0, t)=\mu(t), u(l, t)=\nu(t)$ на двух концах $x=0$ и $x=l$;

$$
\int_{0}^{T} \mu^{2}(t) d t
$$

в случае граничного управления упругой силой $u_{x}(0, t)=\mu(t)$ на одном конще $x=0$;

$$
\int_{0}^{T}\left\{\mu^{2}(t)+\nu^{2}(t)\right\} d t
$$

в случае граничных управлений упругими силами $u_{x}(0, t)=\mu(t), u_{x}(l, t)=\nu(t)$ на двух конщах $x=0$ и $x=l$;

$$
\int_{0}^{T}\left\{\mu^{2}(t)+\left[\nu^{\prime}(t)\right]^{2}\right\} d t
$$

в случае комбинированного граничного управления упругой силой $u_{x}(0, t)=\mu(t)$ на одном конце $x=0$ и смешением $u(l, t)=\nu(t)$ на втором конце $x=l$.

Нами будет установлено, что для ряда задач оптимальные граничные управления зависят не от самих начальных функций $\varphi(x)$ и $\psi(x)$ из начальных условий (2) и финальных функций $\widehat{\varphi}(x)$ и $\widehat{\psi}(x)$ из финальных условий $(3)$, а только от разностей

$$
\widetilde{\varphi}(x)=\widehat{\varphi}(x)-\varphi(x) \text { и } \widetilde{\psi}(x)=\widehat{\psi}(x)-\psi(x)
$$

финального и начального смещений и финальной и начальной скоростей. 
Для получения более изяшных результатов мы будем для задач с граничными условиями одного рода на концах $x=0$ и $x=l$ считать $T$ кратным $2 l$ и равным $T=$ $2 l(n+1)$, где $n=0,1,2, \ldots$, а для задач с граничньми условиями разных родов на концах $x=0$ и $x=l$ считать $T$ кратным $4 l$ и равным $T=4 l(n+1)$, где $n=0,1,2, \ldots$

Наше дальнейшее изложение состоит из восьми пунктов. В каждом из пунктов $1^{\circ}-7^{\circ}$ мы приводим стереотипно получаемые результаты оптимизации одной из семи рассматриваемых нами задач, придерживаясь следующего плана.

1) Установление интегрального тождества, которому при любом $T>0$ должно удовлетворять обобщенное из класса $\widehat{W}_{2}^{1}\left(Q_{T}\right)$ решение $u(x, t)$ смешанной задачи для волнового уравнения (1) с начальными условиями (2) и с рассматриваемьми в данной задаче граничными условиями. (Сразу же отметим, что из этого тождества в силу схемы, изложенной в $[3 ;$ гл. $2, \S 9]$, будет вытекать, что для любого $T>0$ указанная смешанная задача может иметь только одно решение из класса $\widehat{W}_{2}^{1}\left(Q_{T}\right)$.)

2) Построение при $T>l$ в случае граничного управления на двух концах и при $T>2 l$ в случае граничного управления на одном конще специального решения $\widetilde{u}(x, t)$ из класса $\widehat{W}_{2}^{1}\left(Q_{T}\right)$ смешанной задачи для волнового уравнения $\widetilde{u}_{t t}(x, t)-\widetilde{u}_{x x}(x, t)=0$ с начальньми условиями $\widetilde{u}(x, 0)=\varphi(x), \widetilde{u}_{t}(x, 0)=\psi(x)$ и с явно заданньми граничными условиями рассматриваемого в данной задаче типа.

3) Построение при $T=2 l(n+1)$, где $n=0,1,2, \ldots$ (в случае граничных условий на двух концах одного рода), и при $T=4 l(n+1)$, где $n=0,1,2, \ldots$ (в случае граничных условий на двух концах разных родов), явного аналитического вида решения $\widehat{u}(x, t)$ из класса $\widehat{W}_{2}^{1}\left(Q_{T}\right)$ смешанной задачи для волнового уравнения $\widehat{u}_{t t}(x, t)-\widehat{u}_{x x}(x, t)=0$ с нулевьми начальными условиями $\widehat{u}(x, 0)=0, \widehat{u}_{t}(x, 0)=0$ и с произвольно заданными граничными условиями рассматриваемого в данной задаче типа.

4) Установление с помощью результатов предыдущего шага условий связи, которые следует присоединить к интегралу граничной энергии при его минимизации в рассматриваемой задаче граничного управления.

5) Явньй аналитический вид оптимальных граничных управлений для рассматриваемой задачи.

6) Анализ основных свойств найденных оптимальных граничных управлений.

В п. $8^{\circ}$ приводится реализация указанного плана для одной из семи рассматриваемых задач - для задачи 7 об оптимизации комбинированного граничного управления упругой силой на одном конце и смешением на другом конце.

\section{$1^{\circ}$. Задача 1 (об оптимизации граничного управления смещением на одном конце при закрепленном втором конце)}

1) При любом $T>0$ обобщенное из класса $\widehat{W}_{2}^{1}\left(Q_{T}\right)$ решение $u(x, t)$ смешанной задачи для волнового уравнения (1) с начальными условиями $(2)$ из классов $(4)$ и с граничными условиями $u(0, t)=\mu(t), u(l, t)=0$, в которых $\mu(t) \in W_{2}^{1}[0, T]$ и выполнены условия согласования $\varphi(0)=\mu(0), \varphi(l)=0$, определяется как функция $u(x, t)$ 
из класса $\widehat{W}_{2}^{1}\left(Q_{T}\right)$, удовлетворяющая интегральному тождеству

$$
\begin{gathered}
\int_{0}^{l} \int_{0}^{T} u(x, t)\left[\Phi_{t t}(x, t)-\Phi_{x x}(x, t)\right] d x d t+\int_{0}^{l} \varphi(x) \Phi_{t}(x, 0) d x \\
\quad-\int_{0}^{l} \psi(x) \Phi(x, 0) d x-\int_{0}^{T} \mu(t) \Phi_{x}(0, t) d t=0
\end{gathered}
$$

для любой функции $\Phi(x, t)$ из класса $C^{(2)}\left(\bar{Q}_{T}\right)$, удовлетворяющей следуюшим условиям:

$$
\begin{gathered}
\Phi(x, T) \equiv 0, \quad \Phi_{t}(x, T) \equiv 0 \text { при } 0 \leqslant x \leqslant l \\
\Phi(0, t) \equiv 0, \quad \Phi(l, t) \equiv 0 \text { при } 0 \leqslant t \leqslant T .
\end{gathered}
$$

2) При $T>2 l$ функция $\widetilde{u}(x, t)$, определяемая равенством

$$
\widetilde{u}(x, t)=\left\{\begin{array}{lc}
\frac{1}{2}\left[\varphi(x+t)+\varphi(x-t)+\int_{x-t}^{x+t} \psi(\xi) d \xi\right] & \text { в } \Delta_{1}, \\
\frac{1}{2}\left[\varphi(x+t)+\varphi(0)+\int_{0}^{x+t} \psi(\xi) d \xi\right] & \text { в } \Delta_{2}, \\
\frac{1}{2}\left[\varphi(0)+\varphi(2 l)+\int_{0}^{2 l} \psi(\xi) d \xi\right]=C_{0}=\text { const } & \text { в } \Delta_{3},
\end{array}\right.
$$

в котором $\Delta_{1}$ - треугольник, ограниченньй отрезками прямых $x-t=0, x-l=0$ и $t=0, \Delta_{2}$ - треугольник, ограниченный отрезками прямых $x-t=0, x+t-2 l=0$ и $x=0, \Delta_{3}$ - четырехугольник, ограниченньй отрезками прямых $x+t-2 l=0$, $x=0, x-l=0$ и $t-T=0$, при условии, что функции $\varphi(x)$ и $\psi(x)$ продолжены нечетно ${ }^{1}$ относительно точки $x=l$ с сегмента $[0, l]$ на сегмент $[l, 2 l]$ и что вьполнено условие согласования $\varphi(l)=0$, является единственным решением из класса $\widehat{W}_{2}^{1}\left(Q_{T}\right)$ смешанной задачи для волнового уравнения $\widetilde{u}_{t t}(x, t)-\widetilde{u}_{x x}(x, t)=0$ с начальными условиями $\widetilde{u}(x, 0)=\varphi(x), \widetilde{u}_{t}(x, 0)=\psi(x)$ и с граничными условиями

$$
\begin{gathered}
\widetilde{u}(0, t)=\widetilde{\mu}(t)=\left\{\begin{array}{l}
\frac{1}{2}\left[\varphi(t)+\varphi(0)+\int_{0}^{t} \psi(\xi) d \xi\right] \text { при } 0 \leqslant t \leqslant 2 l, \\
0
\end{array}\right. \\
\widetilde{u}(l, t)=\widetilde{\nu}(t)=0 \text { пли } 2 l \leqslant t \leqslant T,
\end{gathered}
$$

3) При $T=2 l(n+1)$, где $n=0,1,2, \ldots$, функция $\widehat{u}(x, t)=u(x, t)-\widetilde{u}(x, t)$, являюшаяся единственным решением из класса $\widehat{W}_{2}^{1}\left(Q_{T}\right)$ волнового уравнения $\widehat{u}_{t t}(x, t)-$ $\widehat{u}_{x x}(x, t)=0$ с нулевьми начальньми условиями $\widehat{u}(x, 0)=0, \widehat{u}_{t}(x, 0)=0$ и с граничными условиями $\widehat{u}(0, t)=\widehat{\mu}(t)=\mu(t)-\widetilde{\mu}(t), \widehat{u}(l, t)=0$ при вьполнении условия $\widehat{\mu}(0)=0$, определяется равенством

$$
\widehat{u}(x, t)=\sum_{k=0}^{n} \underline{\widehat{\mu}}(t-x-2 k l)-\sum_{k=1}^{n+1} \underline{\widehat{\mu}}(t+x-2 k l),
$$

\footnotetext{
${ }^{1}$ При таком продолжении постоянная $C_{0}$ в $(17)$ равна нулю.
} 
в котором через $\widehat{\mu}(t)$ обозначена функция, совпадаюшая с $\widehat{\mu}(t)$ при $0 \leqslant t \leqslant T$ и равная нулю при $t<0$.

4) При $T=2 l(n+1)$, где $n=0,1,2, \ldots$, из равенства (18) и из финальньх условий $(3)$, в которых $\widehat{\varphi}(l)=0$, в предположении, что функции $\widehat{\varphi}(x)$ и $\widehat{\psi}(x)$ также продолжены нечетно относительно точки $x=l$ с сегмента $[0, l]$ на сегмент $[l, 2 l]$, мы получим следующее условие связи

$$
-\sum_{k=0}^{n} \mu^{\prime}(2 l k+x)=\frac{1}{2}\left[\widetilde{\varphi}^{\prime}(x)+\widetilde{\psi}(x)\right]
$$

являющееся равенством элементов $L_{2}[0,2 l]$ и зависящее только от разностей $\widetilde{\varphi}(x)=$ $\widehat{\varphi}(x)-\varphi(x)$ и $\widetilde{\psi}(x)=\widehat{\psi}(x)-\psi(x)$ финального и начального смещений и финальной и начальной скоростей.

5) Решая задачу об отыскании минимума интеграла граничной энергии (8) с условием связи (19), мы получим следуюшее выражение для оптимального граничного управления $u(0, t)=\mu(t)$ :

$$
\mu(t)=L(t)+\alpha(t)
$$

в котором главньй член $L(t)$ является линейной функцией вида

$$
L(t)=\widehat{\varphi}(0) \frac{t}{T}+\varphi(0)\left(1-\frac{t}{T}\right)
$$

а добавочный член $\alpha(t)$ является на сегменте $[0, T]=[0,2 l(n+1)]$ периодической функцией периода $2 l$ и для любого $k=0,1, \ldots, n$ и любого $x$ из сегмента $[0,2 l]$ имеет вид

$$
\alpha(2 l k+x)=\frac{1}{2(n+1)}\left\{\widetilde{\varphi}(0)\left(1-\frac{x}{l}\right)-\widetilde{\varphi}(x)-\int_{0}^{x} \widetilde{\psi}(\xi) d \xi\right\}
$$

6) Подчеркнем, что главньй член (21) является единственной линейной функцией, обрашающейся в $\varphi(0)$ при $t=0$ и в $\widehat{\varphi}(0)$ при $t=T$.

Подчеркнем, что добавочньй член $\alpha(t)$ зависит только от разностей $\widetilde{\varphi}(x)$ и $\widetilde{\psi}(x)$ финального и начального смешений и финальной и начальной скоростей, обрашается в нуль на концах сегментов периодичности (т.е. в точках $0,2 l, 4 l, \ldots, 2 l(n+1))$, равномерно на всем сегменте $[0, T]$ имеет порядок $O\left(\frac{1}{T}\right)$, а в центрах сегментов периодичности (т.е. в точках $l, 3 l, 5 l, \ldots, 2 l n+l)$ принимает значение $-\frac{1}{2(n+1)} \int_{0}^{l} \widetilde{\psi}(\xi) d \xi$, зависящее только от разности $\widetilde{\psi}(x)=\widehat{\psi}(x)-\psi(x)$ финальной и начальной скоростей и совсем не зависящее от начального и от финального смещений.

\section{$2^{\circ}$. Задача 2 (оптимизация граничного управления смещением на двух концах)}

1) При любом $T>0$ обобшенное из класса $\widehat{W}_{2}^{1}\left(Q_{T}\right)$ решение $u(x, t)$ смешанной задачи для волнового уравнения (1) с начальными условиями (2) из классов (4) и с граничными условиями $u(0, t)=\mu(t), u(l, t)=\nu(t)$ из классов (6) при вьполнении 
условий согласования $\varphi(0)=\mu(0), \varphi(l)=\nu(0)$ определяется как функция $u(x, t)$ из класса $\widehat{W}_{2}^{1}\left(Q_{T}\right)$, удовлетворяющая интегральному тождеству

$$
\begin{aligned}
\int_{0}^{l} & \int_{0}^{T} u(x, t)\left[\Phi_{t t}(x, t)-\Phi_{x x}(x, t)\right] d x d t+\int_{0}^{l} \varphi(x) \Phi_{t}(x, 0) d x \\
& \quad-\int_{0}^{l} \psi(x) \Phi(x, 0) d x-\int_{0}^{T} \mu(t) \Phi_{x}(0, t) d t+\int_{0}^{T} \nu(t) \Phi_{x}(l, t) d t=0
\end{aligned}
$$

для любой функции $\Phi(x, t)$ из класса $C^{(2)}\left(\bar{Q}_{T}\right)$, удовлетворяюшей тем же условиям (15) и (16), что и в задаче 1.

2) При $T>l$ функция $\widetilde{u}(x, t)$, определяемая равенством

$$
\widetilde{u}(x, t)= \begin{cases}\frac{1}{2}\left[\varphi(x+t)+\varphi(x-t)+\int_{x-t}^{x+t} \psi(\xi) d \xi\right] & \text { в } \Delta_{1}, \\ \frac{1}{2}\left[\varphi(x+t)+\varphi(0)+\int_{0}^{x+t} \psi(\xi) d \xi\right] & \text { в } \Delta_{2}, \\ \frac{1}{2}\left[\varphi(l)+\varphi(x-t)+\int_{x-t}^{l} \psi(\xi) d \xi\right] & \text { в } \Delta_{3}, \\ \frac{1}{2}\left[\varphi(0)+\varphi(l)+\int_{0}^{l} \psi(\xi) d \xi\right]=C_{0}=\text { const } & \text { в } \Delta_{4},\end{cases}
$$

в котором $\Delta_{1}$ - треугольник, ограниченный отрезками прямых $x-t=0, x+t-l=0$ и $t=0, \Delta_{2}$ - треугольник, ограниченньй отрезками прямых $x-t=0, x+t-l=0$ и $x=0, \Delta_{3}-$ треугольник, ограниченньй отрезками прямых $x-t=0, x+t-l=0$ и $x-l=0, \Delta_{4}$-пятиугольник, ограниченный отрезками прямых $x-t=0, x+t-l=0$, $x=0, x-l=0$ и $t-T=0$, является единственным решением из класса $\widehat{W}_{2}^{1}\left(Q_{T}\right)$ смешанной задачи для волнового уравнения $\widetilde{u}_{t t}(x, t)-\widetilde{u}_{x x}(x, t)=0$ с начальными условиями $\widetilde{u}(x, 0)=\varphi(x), \widetilde{u}_{t}(x, 0)=\psi(x)$ и с граничными условиями

$$
\begin{aligned}
& \widetilde{u}(0, t)=\widetilde{\mu}(t)= \begin{cases}\frac{1}{2}\left[\varphi(t)+\varphi(0)+\int_{0}^{t} \psi(\xi) d \xi\right] & \text { при } 0 \leqslant t \leqslant l, \\
C_{0} & \text { при } l \leqslant t \leqslant T,\end{cases} \\
& \widetilde{u}(l, t)=\widetilde{\nu}(t)=\left\{\begin{array}{lr}
\frac{1}{2}\left[\varphi(l-t)+\varphi(l)+\int_{l-t}^{l} \psi(\xi) d \xi\right] & \text { при } 0 \leqslant t \leqslant l, \\
C_{0} & \text { при } l \leqslant t \leqslant T .
\end{array}\right.
\end{aligned}
$$

$3)$ При $T=2 l(n+1)$, где $n=0,1,2, \ldots$, функция $\widehat{u}(x, t)=u(x, t)-\widetilde{u}(x, t)$, являюшаяся единственньм решением из класса $\widehat{W}{ }_{2}^{1}\left(Q_{T}\right)$ смешанной задачи для волнового уравнения $\widehat{u}_{t t}(x, t)-\widehat{u}_{x x}(x, t)=0$ с нулевыми начальными условиями $\widehat{u}(x, 0)=0$, $\widehat{u}_{t}(x, 0)=0$ и с граничными условиями $\widehat{u}(0, t)=\widehat{\mu}(t)=\mu(t)-\widetilde{\mu}(t), \widehat{u}(l, t)=\widehat{\nu}(t)=$ $\nu(t)-\widetilde{\nu}(t)$, удовлетворяющими условиям согласования $\widehat{\mu}(0)=0, \widehat{\nu}(0)=0$, определяется равенством

$$
\begin{aligned}
\widehat{u}(x, t)= & \sum_{k=0}^{n} \underline{\widehat{\mu}}(t-x-2 k l)-\sum_{k=1}^{n+1} \underline{\widehat{\mu}}(t+x-2 k l) \\
& +\sum_{k=0}^{n} \underline{\widehat{\nu}}(t+x-2 k l-l)-\sum_{k=1}^{n+1} \underline{\widehat{\nu}}(t-x-2 k l+l),
\end{aligned}
$$


в котором символы $\underline{\hat{\mu}}(t)$ и $\underline{\underline{\nu}}(t)$ обозначают функции, совпадаюшие с $\widehat{\mu}(t)$ и $\widehat{\nu}(t)$ соответственно при $0 \leqslant \bar{t} \leqslant T$ и равные нулю при $t<0$.

4) Из равенства (25) и финальных условий (3) и из условия согласования получим следуюшие три зависяшие только от разностей (13) условия связи:

$$
\left\{\begin{array}{l}
-\sum_{k=0}^{n} \mu^{\prime}(2 l k+x)+\sum_{k=1}^{n+1} \nu^{\prime}(2 l k+x-l)=\frac{1}{2}\left[\widetilde{\varphi}^{\prime}(x)+\widetilde{\psi}(x)\right], \\
-\sum_{k=1}^{n+1} \mu^{\prime}(2 l k-x)+\sum_{k=0}^{n} \nu^{\prime}(2 l k+l-x)=\frac{1}{2}\left[\widetilde{\varphi}^{\prime}(x)-\widetilde{\psi}(x)\right], \\
\int_{0}^{T}\left[\mu^{\prime}(t)+\nu^{\prime}(t)\right] d t=\widetilde{\varphi}(0)+\widetilde{\varphi}(l),
\end{array}\right.
$$

первые два из которых являются равенствами элементов $L_{2}[0, l]$.

5) Решая задачу об отыскании минимума интеграла граничной энергии (9) с условиями связи (26), мы получим следуюшие выражения для оптимальных граничных управлений $u(0, t)=\mu(t)$ и $u(l, t)=\nu(t)$ :

$$
\mu(t)=L_{1}(t)+\alpha_{1}(t), \quad \nu(t)=L_{2}(t)+\alpha_{2}(t),
$$

главные члены $L_{1}(t)$ и $L_{2}(t)$ которых являются линейными функциями вида

$$
L_{1}(t)=\widehat{\varphi}(0) \frac{t}{T}+\varphi(0)\left(1-\frac{t}{T}\right), \quad L_{2}(t)=\widehat{\varphi}(l) \frac{t}{T}+\varphi(l)\left(1-\frac{t}{T}\right),
$$

а добавочные члены $\alpha_{1}(t)$ и $\alpha_{2}(t)$ которых являются на сегменте $[0, T]=[0,2 l(n+1)]$ периодическими функциями периода $2 l$ и для любого $k=0,1, \ldots, n$ и любого $x$ из сегмента $0 \leqslant x \leqslant 2 l$ имеют вид

$$
\begin{aligned}
& \alpha_{1}(2 l k+x)=\frac{\widetilde{\varphi}(l)-\widetilde{\varphi}(0)}{4 l(n+1)} x \\
& +\left\{\begin{array}{lr}
\frac{1}{4(n+1)}\left[\widetilde{\varphi}(0)-\widetilde{\varphi}(x)-\int_{0}^{x} \widetilde{\psi}(\xi) d \xi\right] \\
\frac{1}{4(n+1)}\left[\widetilde{\varphi}(0)-2 \widetilde{\varphi}(l)+\widetilde{\varphi}(2 l-x)-\int_{0}^{2 l-x} \widetilde{\psi}(\xi) d \xi\right] & \text { при } 0 \leqslant x \leqslant l,
\end{array}, \quad \text { при } 0 \leqslant x \leqslant l,\right. \\
& \alpha_{2}(2 l k+x)=\frac{\widetilde{\varphi}(0)-\widetilde{\varphi}(l)}{4 l(n+1)} x \\
& +\left\{\begin{array}{l}
\frac{1}{4(n+1)}\left[\widetilde{\varphi}(l)-\widetilde{\varphi}(l-x)-\int_{l-x}^{l} \widetilde{\psi}(\xi) d \xi\right] \\
\frac{1}{4(n+1)}\left[\widetilde{\varphi}(l)-2 \widetilde{\varphi}(0)+\widetilde{\varphi}(x-l)-\int_{x-l}^{l} \widetilde{\psi}(\xi) d \xi\right] \text { при } l \leqslant x \leqslant 2 l .
\end{array}\right.
\end{aligned}
$$

6) Подчеркнем, что первьй из главных членов (28) является единственной линейной функцией, обрашающейся в $\varphi(0)$ при $t=0$ и в $\widehat{\varphi}(0)$ при $t=T$, а второй из главных членов $(28)$ является единственной линейной функцией, обрашающейся в $\varphi(l)$ при $t=0$ и в $\widehat{\varphi}(l)$ при $t=T$. 
Подчеркнем, что добавочные члены $(29)$ и $(30)$ зависят только от разностей $\widetilde{\varphi}(x)$ и $\widetilde{\psi}(x)$ финального и начального смещений и финальной и начальной скоростей, обращаются в нуль на концах сегментов периодичности (т.е. в точках $0,2 l, 4 l, 6 l, \ldots, 2 l(n+1))$, равномерно на всем сегменте $[0, T]$ имеют порядок $O\left(\frac{1}{T}\right)$, а в центрах сегментов периодичности (т.е. в точках $l, 3 l, 5 l, \ldots, 2 l n+l)$ принимают значение $-\frac{1}{4(n+1)} \int_{0}^{l} \widetilde{\psi}(\xi) d \xi$, зависяшее только от разности $\widetilde{\psi}(x)=\widehat{\psi}(x)-\psi(x)$ финальной и начальной скоростей и совсем не зависящее от начального и от финального смещений.

\section{$3^{\circ}$. Задача 3 (оптимизация граничного управления смешением на одном конце при свободном втором конце)}

1) При любом $T>0$ обобщенное из класса $\widehat{W}_{2}^{1}\left(Q_{T}\right)$ решение $u(x, t)$ смешанной задачи для волнового уравнения (1) с начальными условиями $(2)$ из классов $(4)$ и с граничными условиями $u(0, t)=\mu(t), u_{x}(l, t)=0$, в которых $\mu(t) \in W_{2}^{1}[0, T]$, определяется как функция $u(x, t)$ из класса $\widehat{W}_{2}^{1}\left(Q_{T}\right)$, удовлетворяющая тому же самому тождеству $(14)$, что и в задаче 1, но для любой функции $\Phi(x, t)$ из класса $C^{(2)}\left(\bar{Q}_{T}\right)$, для которой выполнены условия (15) и условия $\Phi(0, t) \equiv 0, \Phi_{x}(l, t) \equiv 0$ при $0 \leqslant t \leqslant T$.

2) При $T>2 l$ функция $\widetilde{u}(x, t)$, определяемая равенством (17) с теми же $\Delta_{1}, \Delta_{2}$ и $\Delta_{3}$, что и в задаче 1 , при условии, что функщии $\varphi(x)$ и $\psi(x)$ продолжены четно относительно точки $x=l$ с сегмента $[0, l]$ на сегмент $[l, 2 l]$, является единственным решением из класса $\widehat{W}_{2}^{1}\left(Q_{T}\right)$ смешанной задачи для волнового уравнения $\widetilde{u}_{t t}(x, t)-\widetilde{u}_{x x}(x, t)=0$ с начальньми условиями $\widetilde{u}(x, 0)=\varphi(x), \widetilde{u}_{t}(x, 0)=\psi(x)$ и с граничньми условиями

$$
\begin{gathered}
\widetilde{u}(0, t)=\widetilde{\mu}(t)=\left\{\begin{array}{l}
\frac{1}{2}\left[\varphi(t)+\varphi(0)+\int_{0}^{t} \psi(\xi) d \xi\right] \quad \text { при } 0 \leqslant t \leqslant 2 l, \\
\varphi(0)+\int_{0}^{l} \psi(\xi) d \xi=C_{0}=\mathrm{const} \text { при } 2 l<t \leqslant T,
\end{array}\right. \\
\widetilde{u}_{x}(l, t)=0 \text { как элемент пространства } L_{2}[0, T] .
\end{gathered}
$$

3) При $T=4 l(n+1)$, где $n=0,1,2, \ldots$, функция $\widehat{u}(x, t)=u(x, t)-\widetilde{u}(x, t)$, являюшаяся единственньм решением из класса $\widehat{W}_{2}^{1}\left(Q_{T}\right)$ смешанной задачи для волнового уравнения $\widehat{u}_{t t}(x, t)-\widehat{u}_{x x}(x, t)=0$ с нулевыми начальными условиями $\widehat{u}(x, 0)=0$, $\widehat{u}_{t}(x, 0)=0$ и с граничными условиями $\widehat{u}(0, t)=\widehat{\mu}(t)=\mu(t)-\widetilde{\mu}(t), \widehat{u}_{x}(l, t)=0$, удовлетворяюшими условию согласования $\widehat{\mu}(0)=0$, определяется равенством

$$
\widehat{u}(x, t)=\sum_{k=0}^{2 n+1}(-1)^{k} \underline{\widehat{\mu}}(t-x-2 k l)-\sum_{k=1}^{2 n+2}(-1)^{k} \underline{\widehat{\mu}}(t+x-2 k l),
$$

в котором символ $\underline{\widehat{\mu}}(t)$ обозначает функцию, совпадающую с $\widehat{\mu}(t)$ при $0 \leqslant t \leqslant T$ и равную нулю при $t<0$.

4) Из равенства (31) и финальных условий (3) и из условия согласования в предположении, что функции $\widehat{\varphi}(x)$ и $\widehat{\psi}(x)$ также продолжены четно относительно точки 
$x=l$ с сегмента $[0, l]$ на сегмент $[l, 2 l]$, мы получим следуюшие зависяшие только от разностей (13) условия связи:

$$
\left\{\begin{array}{r}
-\sum_{k=0}^{2 n+1}(-1)^{k} \mu^{\prime}(2 l k+x)=\frac{1}{2}\left[\widetilde{\varphi}^{\prime}(x)+\widetilde{\psi}(x)\right] \\
\int_{0}^{T} \mu^{\prime}(t) d t=\widetilde{\varphi}(0),
\end{array}\right.
$$

первое из которых является равенством элементов $L_{2}[0,2 l]$.

5) Решая задачу об отыскании минимума интеграла граничной энергии (8) с условиями связи (32), мы получим следующее выражение для оптимального граничного управления $u(0, t)=\mu(t)$ :

$$
\mu(t)=L(t)+\alpha(t)
$$

главный член $L(t)$ которого определяется тем же равенством (21), что и в задаче 1 , а добавочный член $\alpha(t)$ которого является на сегменте $[0, T]=[0,4 l(n+1)]$ периодической функцией периода $4 l$ и для любого $k=0,1, \ldots, n$ и любого $x$ из сегмента $[0,4 l]$ равен

$$
\alpha(4 l k+x)= \begin{cases}\frac{1}{4(n+1)}\left[\widetilde{\varphi}(0)-\widetilde{\varphi}(x)-\int_{0}^{x} \widetilde{\psi}(\xi) d \xi\right] & \text { при } 0 \leqslant x \leqslant 2 l, \\ \frac{1}{4(n+1)}\left[\widetilde{\varphi}(x-2 l)-\widetilde{\varphi}(0)-\int_{x-2 l}^{2 l} \widetilde{\psi}(\xi) d \xi\right] & \text { при } 2 l \leqslant x \leqslant 4 l .\end{cases}
$$

6) Подчеркнем, что (как и в задачах 1 и 2 ) главньй член $L(t)$ является единственной линейной функцией, обрашаюшейся в $\varphi(0)$ при $t=0$ и обрашаюшейся в $\widehat{\varphi}(0)$ при $t=T$.

Подчеркнем, что добавочный член $\alpha(t)$, определяемый равенством (34), зависит только от разностей $\widetilde{\varphi}(x)$ и $\widetilde{\psi}(x)$ финального и начального смещений и финальной и начальной скоростей, обрашается в нуль на концах сегментов периодичности (т.е. в точках $0,4 l, 8 l, \ldots, 4 l(n+1))$, равномерно на всем сегменте $[0, T]$ имеет порядок $O\left(\frac{1}{T}\right)$, а в центрах сегментов периодичности (т.е. в точках $2 l, 6 l, 10 l, \ldots, 4 l n+2 l)$ принимает значение $-\frac{1}{2(n+1)} \int_{0}^{l} \widetilde{\psi}(\xi) d \xi$, зависяшее только от разности $\widetilde{\psi}(x)=\widehat{\psi}(x)-\psi(x)$ финальной и начальной скоростей и совсем не зависящее от начального и от финального смешений.

\section{$4^{\circ}$. Задача 4 (оптимизация граничного управления упругой силой на одном конце при закрепленном другом конце)}

1) При любом $T>0$ обобшенное из класса $\widehat{W}_{2}^{1}\left(Q_{T}\right)$ решение $u(x, t)$ волнового уравнения (1) с начальными условиями (2) из классов (4) и с граничными условиями $u_{x}(0, t)=\mu(t), u(l, t)=0$, в которых $\mu(t) \in L_{2}[0, T]$ и выполнено условие согласования $\varphi(l)=0$, определяется как функция $u(x, t)$ из класса $\widehat{W}_{2}^{1}\left(Q_{T}\right)$, которая удовлетворяет интегральному тождеству

$$
\begin{gathered}
\int_{0}^{l} \int_{0}^{T} u(x, t)\left[\Phi_{t t}(x, t)-\Phi_{x x}(x, t)\right] d x d t+\int_{0}^{l} \varphi(x) \Phi_{t}(x, 0) d x \\
\quad-\int_{0}^{l} \psi(x) \Phi(x, 0) d x+\int_{0}^{T} \mu(t) \Phi(0, t) d t=0
\end{gathered}
$$


для любой функции $\Phi(x, t)$ из класса $C^{(2)}\left(\bar{Q}_{T}\right)$, удовлетворяющей условиям $(15)$ и условиям $\Phi_{x}(0, t) \equiv 0, \Phi(l, t) \equiv 0$ при $0 \leqslant t \leqslant T$.

$2)$ При $T>2 l$ функция $\widetilde{u}(x, t)$, определяемая равенством (17) с теми же $\Delta_{1}, \Delta_{2}$ и $\Delta_{3}$, что и в задаче 1 , в предположении, что функции $\varphi(x)$ и $\psi(x)$ продолжены нечетно $^{2}$ относительно точки $x=l$ с сегмента $[0, l]$ на сегмент $[l, 2 l]$ и вьполнено условие согласования $\varphi(l)=0$, является единственным решением из класса $\widehat{W}_{2}^{1}\left(Q_{T}\right)$ смешанной задачи для волнового уравнения $\widetilde{u}_{t t}(x, t)-\widetilde{u}_{x x}(x, t)=0$ с начальными условиями $\widetilde{u}(x, 0)=\varphi(x), \widetilde{u}_{t}(x, 0)=\psi(x)$ и с граничными условиями

$$
\begin{gathered}
\widetilde{u}_{x}(0, t)=\widetilde{\mu}(t)= \begin{cases}\frac{1}{2}\left[\varphi^{\prime}(t)+\psi(t)\right] & \text { при } 0 \leqslant t \leqslant 2 l, \\
0 & \text { при } 2 l<t \leqslant T,\end{cases} \\
\widetilde{u}(l, t)=0 \text { при } 0 \leqslant t \leqslant T .
\end{gathered}
$$

3) При $T=4 l(n+1)$, где $n=0,1,2, \ldots$, функция $\widehat{u}(x, t)=u(x, t)-\widetilde{u}(x, t)$, являюшаяся единственным решением из класса $\widehat{W}_{2}^{1}\left(Q_{T}\right)$ смешанной задачи для волнового уравнения $\widehat{u}_{t t}(x, t)-\widehat{u}_{x x}(x, t)=0$ с нулевыми начальными условиями $\widehat{u}(x, 0)=0$, $\widehat{u}_{t}(x, 0)=0$ и с граничными условиями $\widehat{u}_{x}(0, t)=\widehat{\mu}(t)=\mu(t)-\widetilde{\mu}(t), \widehat{u}(l, t)=0$, определяется равенством

$$
\widehat{u}(x, t)=-\sum_{k=0}^{2 n+1}(-1)^{k} \int_{0}^{t-x-2 k l} \underline{\widehat{\mu}}(\tau) d \tau-\sum_{k=1}^{2 n+2}(-1)^{k} \int_{0}^{t+x-2 k l} \widehat{\widehat{\mu}}(\tau) d \tau,
$$

в котором символ $\widehat{\mu}(t)$ обозначает функцию, совпадающую с $\widehat{\mu}(t)$ при $0 \leqslant t \leqslant T$ и равную нулю при $t<0$.

4) Из равенства (36) и финальных условий (3) в предположении, что $\widehat{\varphi}(l)=0$ и функции $\widehat{\varphi}(x)$ и $\widehat{\psi}(x)$ также продолжены нечетно относительно точки $x=l$ с сегмента $[0, l]$ на сегмент $[l, 2 l]$, мы получим следующее зависящее только от разностей (13) условие связи:

$$
-\sum_{k=0}^{2 n+1}(-1)^{k} \mu(2 l k+x)=\frac{1}{2}\left[\widetilde{\varphi}^{\prime}(x)+\widetilde{\psi}(x)\right]
$$

являюшееся равенством элементов $L_{2}[0,2 l]$.

5) Решая задачу об отыскании минимума интеграла граничной энергии (10) с условием связи (37), мы получим, что оптимальное граничное управление $u_{x}(0, t)=\mu(t)$ является на сегменте $[0, T]=[0,4 l(n+1)]$ периодической функцией периода $4 l$ и для любого $k=0,1, \ldots, n$ и любого $x$ из полусегмента $0 \leqslant x<4 l$ определяется равенст$\mathrm{BOM}^{3}$

$$
\mu(4 l k+x)= \begin{cases}-\frac{1}{4(n+1)}\left[\widetilde{\varphi}^{\prime}(x)+\widetilde{\psi}(x)\right] & \text { при } 0 \leqslant x \leqslant 2 l, \\ \frac{1}{4(n+1)}\left[\widetilde{\varphi}^{\prime}(x-2 l)+\widetilde{\psi}(x-2 l)\right] & \text { при } 2 l<x<4 l .\end{cases}
$$

\footnotetext{
${ }^{2}$ При таком продолжении постоянная $C_{0}$ в $(17)$ равна нулю.

${ }^{3}$ Приводимое равенство не определяет $\mu(t)$ в точке $t=T$, но поскольку $\mu(t)$ является элементом $L_{2}[0, T]$, ее значение в одной точке $t=T$ может быть любым.
} 
6) Подчеркнем, что оптимальная граничная сила $\mu(t)$ зависит только от разностей $\widetilde{\varphi}(x)$ и $\widetilde{\psi}(x)$ финального и начального смешений и финальной и начальной скоростей и что для квадрата нормы $\mu(t)$ в $L_{2}[0, T]$ справедлива оценка

$$
\int_{0}^{T} \mu^{2}(t) d t=O\left(\frac{1}{T}\right)
$$

Отметим еще одно важное свойство $\mu(t)$ : интеграл от $\mu(t)$ по любому содержащемуся в $[0, T]=[0,4 l(n+1)]$ сегменту длины $4 l$ равен нулю.

\section{$5^{\circ}$. Задача 5 (оптимизация граничного управления упругой силой на одном конце при свободном втором конце)}

1) При любом $T>0$ обобшенное из класса $\widehat{W}_{2}^{1}\left(Q_{T}\right)$ решение $u(x, t)$ смешанной задачи для волнового уравнения (1) с начальными условиями (2) из классов (4) и с граничными условиями $u_{x}(0, t)=\mu(t), u_{x}(l, t)=0$, в которых $\mu(t) \in L_{2}[0, T]$, определяется как функция $u(x, t)$ из класса $\widehat{W}_{2}^{1}\left(Q_{T}\right)$, удовлетворяюшая тому же интегральному тождеству (35), что и в задаче 4 , для любой функции $\Phi(x, t)$ из класса $C^{(2)}\left(\bar{Q}_{T}\right)$, удовлетворяющей условиям (15) и условиям $\Phi_{x}(0, t) \equiv 0, \Phi_{x}(l, t) \equiv 0$ при $0 \leqslant t \leqslant T$.

$2)$ При $T>2 l$ функция $\widetilde{u}(x, t)$, определяемая тем же равенством (17) с теми же $\Delta_{1}$, $\Delta_{2}$ и $\Delta_{3}$, что и в задаче 1 , при условии, что функции $\varphi(x)$ и $\psi(x)$ продолжены четно относительно точки $x=l$ с сегмента $[0, l]$ на сегмент $[l, 2 l]$, является единственным решением из класса $\widehat{W}_{2}^{1}\left(Q_{T}\right)$ смешанной задачи для волнового уравнения $\widetilde{u}_{t t}(x, t)-$ $\widetilde{u}_{x x}(x, t)=0$ с начальными условиями $\widetilde{u}(x, 0)=\varphi(x), \widetilde{u}_{t}(x, 0)=\psi(x)$ и с граничными условиями

$$
\begin{gathered}
\widetilde{u}_{x}(0, t)=\widetilde{\mu}(t)= \begin{cases}\frac{1}{2}\left[\varphi^{\prime}(t)+\psi(t)\right] & \text { при } 0 \leqslant t \leqslant 2 l, \\
0 & \text { при } 2 l<t \leqslant T,\end{cases} \\
\widetilde{u}_{x}(l, t)=0 \text { как элемент пространства } L_{2}[0, T] .
\end{gathered}
$$

3) При $T=2 l(n+1)$, где $n=1,2, \ldots$, функция $\widehat{u}(x, t)=u(x, t)-\widetilde{u}(x, t)$, являюшаяся единственным решением из класса $\widehat{W}_{2}^{1}\left(Q_{T}\right)$ смешанной задачи для волнового уравнения $\widehat{u}_{t t}(x, t)-\widehat{u}_{x x}(x, t)=0$ с нулевыми начальными условиями $\widehat{u}(x, 0)=0$, $\widehat{u}_{t}(x, 0)=0$ и с граничными условиями $\widehat{u}_{x}(0, t)=\widehat{\mu}(t)=\mu(t)-\widetilde{\mu}(t), \widehat{u}_{x}(l, t)=0$, определяется равенством

$$
\widehat{u}(x, t)=-\sum_{k=0}^{n} \int_{0}^{t-x-2 k l} \underline{\widehat{\mu}}(\tau) d \tau-\sum_{k=1}^{n+1} \int_{0}^{t+x-2 k l} \underline{\widehat{\mu}}(\tau) d \tau,
$$

в котором символом $\widehat{\mu}(t)$ обозначена функция, совпадающая с $\widehat{\mu}(t)$ при $0 \leqslant t \leqslant T$ и равная нулю при $t<\overline{0}$.

4) Из равенства (38) и финальных условий (3) в предположении, что функции $\widehat{\varphi}(x)$ и $\widehat{\psi}(x)$ также продолжены четно относительно точки $x=l$ с сегмента $[0, l]$ на сегмент $[l, 2 l]$, мы получим следующие два условия связи:

$$
\left\{\begin{array}{l}
\sum_{k=0}^{n} \mu(2 l k+x)=\frac{1}{2}\left[\widetilde{\varphi}^{\prime}(x)+\widetilde{\psi}(x)\right], \\
-\sum_{k=1}^{n+1} \int_{0}^{2 l k} \mu(t) d t-\sum_{k=1}^{n} \int_{0}^{2 l k} \mu(t) d t=\widetilde{\varphi}(0)-2(n+1) \int_{0}^{l} \psi(x) d x,
\end{array}\right.
$$


первое из которых зависит только от разностей (13) и является равенством элементов $L_{2}[0,2 l]$, а второе зависит от разности смещений $\widetilde{\varphi}(x)$ и от начальной скорости $\psi(x)$.

5) Решая задачу об отыскании минимума интеграла граничной энергии (10) с условиями связи (39), мы получим, что оптимальная упругая граничная сила $u_{x}(0, t)=$ $\mu(t)$ является суммой двух слагаемых

$$
\mu(t)=L(t)+\alpha(t)
$$

первое из которых $L(t)$ при постоянном числе $\widehat{\lambda}$ вида

$$
\widehat{\lambda}=\frac{\widetilde{\varphi}(0)-(n+1) \int_{0}^{l}[\widehat{\psi}(x)+\psi(x)] d x}{\frac{l}{3} n(n+1)(n+2)}
$$

является линейной функцией вида $L(t)=\frac{\widehat{\lambda}}{2 l} t$, а второе из которых $\alpha(t)$ является на сегменте $[0, T]=[0,2 l(n+1)]$ периодической функцией периода $2 l$ и для любого $k=$ $0,1, \ldots, n$ и любого $x$ из полусегмента $0 \leqslant x<2 l$ имеет вид

$$
\alpha(2 l k+x)=-\frac{1}{2(n+1)}\left[\widetilde{\varphi}^{\prime}(x)+\widetilde{\psi}(x)\right]-\widehat{\lambda}\left(\frac{x}{2 l}+\frac{n}{2}\right) .
$$

6) Подчеркнем, что входящая в оптимальную граничную силу $\mu(t)$ линейная функция $L(t)$ равномерно на всем сегменте $[0, T] \equiv[0,2 l(n+1)]$ имеет порядок $O\left(\frac{1}{T}\right)$, а в случае, когда финальная и начальная скорости удовлетворяют условию $\int_{0}^{l}[\widehat{\psi}(x)+\psi(x)] d x=0,-$ более высокий порядок $O\left(\frac{1}{T^{2}}\right)$.

Отметим, кроме того, что квадрат нормы оптимальной граничной силы $\mu(t)$ в пространстве $L_{2}[0, T]$ имеет порядок

$$
\int_{0}^{T} \mu^{2}(t) d t=O\left(\frac{1}{T}\right)
$$

в то время как для функции $\mu(t)$ справедливо равенство

$$
\int_{0}^{T} \mu(t) d t=\int_{0}^{l}[\psi(x)-\widehat{\psi}(x)] d x=-\int_{0}^{l} \widetilde{\psi}(x) d x .
$$

Все указанные в этом пункте результаты с подробными обоснованиями опубликованы в нашей работе [4].

\section{$6^{\circ}$. Задача 6 (оптимизация граничных управлений упругой силой на двух концах)}

1) При любом $T>0$ обобшенное из класса $\widehat{W}_{2}^{1}\left(Q_{T}\right)$ решение $u(x, t)$ смешанной задачи (1) с начальными условиями (2) из классов (4) и с граничными условиями $u_{x}(0, t)=\mu(t), u_{x}(l, t)=\nu(t)$ из класса $(7)$ определяется как функция $u(x, t)$ из класса $\widehat{W_{2}^{1}}\left(Q_{T}\right)$, удовлетворяющая интегральному тождеству

$$
\begin{aligned}
\int_{0}^{l} & \int_{0}^{T} u(x, t)\left[\Phi_{t t}(x, t)-\Phi_{x x}(x, t)\right] d x d t+\int_{0}^{l} \varphi(x) \Phi_{t}(x, 0) d x \\
& -\int_{0}^{l} \psi(x) \Phi(x, 0) d x+\int_{0}^{T} \mu(t) \Phi(0, t) d t-\int_{0}^{T} \nu(t) \Phi(l, t) d t=0
\end{aligned}
$$


для любой функции $\Phi(x, t)$ из класса $C^{(2)}\left(\bar{Q}_{T}\right)$, удовлетворяющей условиям $(15)$ и условиям $\Phi_{x}(0, t) \equiv 0, \Phi_{x}(l, t) \equiv 0$ при $0 \leqslant t \leqslant T$.

2) При $T>l$ функция $\widetilde{u}(x, t)$, определяемая равенством (24) с теми же $\Delta_{1}, \Delta_{2}, \Delta_{3}$ и $\Delta_{4}$, что и в задаче 2 , является единственным решением из класса $\widehat{W}_{2}^{1}\left(Q_{T}\right)$ смешанной задачи для волнового уравнения $\widetilde{u}_{t t}(x, t)-\widetilde{u}_{x x}(x, t)=0$ с начальными условиями $\widetilde{u}(x, 0)=\varphi(x), \widetilde{u}_{t}(x, 0)=\psi(x)$ и с граничными условиями

$$
\begin{aligned}
& \widetilde{u}_{x}(0, t)=\widetilde{\mu}(t)= \begin{cases}\frac{1}{2}\left[\varphi^{\prime}(t)+\psi(t)\right] & \text { при } 0 \leqslant t \leqslant l, \\
0 & \text { при } l<t \leqslant T,\end{cases} \\
& \widetilde{u}_{x}(l, t)=\widetilde{\nu}(t)= \begin{cases}\frac{1}{2}\left[\varphi^{\prime}(l-t)-\psi(l-t)\right] & \text { при } 0 \leqslant t \leqslant l, \\
0 & \text { при } l<t \leqslant T .\end{cases}
\end{aligned}
$$

3) При $T=2 l(n+1)$, где $n=0,1,2, \ldots$, функция $\widehat{u}(x, t)=u(x, t)-\widetilde{u}(x, t)$, являюшаяся единственньм решением из класса $\widehat{W}_{2}^{1}\left(Q_{T}\right)$ смешанной задачи для волнового уравнения $\widehat{u}_{t t}(x, t)-\widehat{u}_{x x}(x, t)=0$ с нулевыми начальными условиями $\widehat{u}(x, 0)=0$, $\widehat{u}_{t}(x, 0)=0$ и с граничными условиями $\widehat{u}_{x}(0, t)=\widehat{\mu}(t)=\mu(t)-\widetilde{\mu}(t), \widehat{u}_{x}(l, t)=\widehat{\nu}(t)=$ $\nu(t)-\widetilde{\nu}(t)$, определяется равенством

$$
\begin{aligned}
\widehat{u}(x, t)= & -\sum_{k=0}^{n} \int_{0}^{t-x-2 k l} \underline{\widehat{\mu}}(\tau) d \tau-\sum_{k=1}^{n+1} \int_{0}^{t+x-2 k l} \underline{\underline{\mu}}(\tau) d \tau \\
& +\sum_{k=0}^{n} \int_{0}^{t+x-l-2 k l} \underline{\widehat{\nu}}(\tau) d \tau+\sum_{k=1}^{n+1} \int_{0}^{t-x+l-2 k l} \widehat{\widehat{v}}(\tau) d \tau,
\end{aligned}
$$

в котором символы $\widehat{\mu}(t)$ и $\underline{\widehat{\nu}}(t)$ обозначают функции, совпадаюшие с $\widehat{\mu}(t)$ и $\widehat{\nu}(t)$ соответственно при $0 \leqslant t \leqslant T$ и равные нулю при $t<0$.

4) Из равенства (41) и финальных условий (3) мы получим следуюшие три условия связи:

$$
\left\{\begin{array}{l}
-\sum_{k=0}^{n} \mu(2 l k+x)+\sum_{k=1}^{n+1} \nu(2 l k-l+x)=\frac{1}{2}\left[\widetilde{\varphi}^{\prime}(x)+\widetilde{\psi}(x)\right], \\
\sum_{k=1}^{n+1} \mu(2 l k-x)-\sum_{k=0}^{n} \nu(2 l k+l-x)=\frac{1}{2}\left[\widetilde{\varphi}^{\prime}(x)-\widetilde{\psi}(x)\right], \\
\sum_{k=1}^{n+1} \int_{0}^{2 l k}[\nu(\tau)-\mu(\tau)] d \tau+\sum_{k=1}^{n} \int_{0}^{2 l k}[\nu(\tau)-\mu(\tau)] d \tau \\
+\sum_{k=1}^{n+1} \int_{0}^{2 l k-l}[\nu(\tau)-\mu(\tau)] d \tau+\sum_{k=0}^{n} \int_{0}^{2 l k+l}[\nu(\tau)-\mu(\tau)] d \tau \\
=\widetilde{\varphi}(0)+\widetilde{\varphi}(l)-4(n+1) \int_{0}^{l} \psi(x) d x,
\end{array}\right.
$$

первые два из которых являются равенствами элементов $L_{2}[0, l]$ и зависят только от разностей (13), а третье зависит от разности смещений $\widetilde{\varphi}(x)$ и от начальной скорости $\psi(x)$. 
5) Решая задачу об отыскании минимума интеграла граничной энергии (11) с тремя условиями связи (42), мы получим, что оптимальные граничные управления $u_{x}(0, t)=\mu(t)$ и $u_{x}(l, t)=\nu(t)$ являются суммами двух слагаемых $\mu(t)=L_{1}(t)+\alpha_{1}(t)$, $\nu(t)=L_{2}(t)+\alpha_{2}(t)$, первые из которых $L_{1}(t)$ и $L_{2}(t)$ при постоянном числе

$$
\widehat{\lambda}=\frac{\frac{1}{2}[\widetilde{\varphi}(0)+\widetilde{\varphi}(l)]-(n+1) \int_{0}^{l}[\widehat{\psi}(x)+\psi(x)] d x}{\frac{1}{3} l(n+1)(2 n+1)(2 n+3)}
$$

являются линейными функциями вида

$$
L_{1}(t)=\frac{\widehat{\lambda}}{l} t, \quad L_{2}(t)=-\frac{\widehat{\lambda}}{l} t
$$

а вторые из которых $\alpha_{1}(t)$ и $\alpha_{2}(t)$ являются на сегменте $[0, T]=[0,2 l(n+1)]$ периодическими функциями периода $2 l$ и для любого $k=0,1, \ldots, n$ и любого $x$ из $[0,2 l)$ имеют вид

$$
\begin{gathered}
\alpha_{1}(2 l k+x)= \begin{cases}-\frac{1}{4(n+1)}\left[\widetilde{\varphi}^{\prime}(x)+\widetilde{\psi}(x)\right]-\widehat{\lambda}\left(\frac{x}{l}+n+\frac{1}{2}\right) & \text { при } 0 \leqslant x<l, \\
\frac{1}{4(n+1)}\left[\widetilde{\varphi}^{\prime}(2 l-x)-\widetilde{\psi}(2 l-x)\right]-\widehat{\lambda}\left(\frac{x}{l}+n-\frac{1}{2}\right) & \text { при } l \leqslant x<2 l,\end{cases} \\
\alpha_{2}(2 l k+x)= \begin{cases}-\frac{1}{4(n+1)}\left[\widetilde{\varphi}^{\prime}(l-x)-\widetilde{\psi}(l-x)\right]+\widehat{\lambda}\left(\frac{x}{l}+n+\frac{1}{2}\right) & \text { при } 0 \leqslant x<l, \\
\frac{1}{4(n+1)}\left[\widetilde{\varphi}^{\prime}(x-l)+\widetilde{\psi}(x-l)\right]+\widehat{\lambda}\left(\frac{x}{l}+n-\frac{1}{2}\right) & \text { при } l \leqslant x<2 l .\end{cases}
\end{gathered}
$$

6) Отметим, что каждая из линейных функций $L_{1}(t)$ и $L_{2}(t)$ равномерно на сегменте $[0, T]=[0,2 l(n+1)]$ имеет порядок $O\left(\frac{1}{T}\right)$, а в случае, когда финальная скорость $\widehat{\psi}(x)$ и начальная скорость $\psi(x)$ связаны соотношением $\int_{0}^{l}[\widehat{\psi}(x)+\psi(x)] d x=0,-$ более высокий порядок $O\left(\frac{1}{T^{2}}\right)$.

Для квадратов норм в $L_{2}[0, T]$ оптимальных граничных управлений $\mu(t)$ и $\nu(t)$ справедливы оценки

$$
\int_{0}^{T} \mu^{2}(t) d t=O\left(\frac{1}{T}\right), \quad \int_{0}^{T} \nu^{2}(t) d t=O\left(\frac{1}{T}\right)
$$

а для самих $\mu(t)$ и $\nu(t)$ справедливы равенства

$$
\int_{0}^{T} \mu(t) d t=-\frac{1}{2} \int_{0}^{l} \widetilde{\psi}(x) d x, \quad \int_{0}^{T} \nu(t) d t=\frac{1}{2} \int_{0}^{l} \widetilde{\psi}(x) d x .
$$

Сумма оптимальных граничных управлений $\mu(t)+\nu(t)$ обладает следующим удивительньм свойством: эта сумма является на сегменте $[0, T]=[0,2 l(n+1)]$ периодической функцией периода $2 l$, причем интеграл от этой суммы по любому содержащемуся в $[0, T]$ сезменту длины $2 l$ равен нулю. 


\section{$7^{\circ}$. Задача 7 (оптимизация комбинированного граничного управления упругой силой на одном конце и смешением на другом конце)}

1) При любом $T>0$ обобщенное решение $u(x, t)$ из класса $\widehat{W}_{2}^{1}\left(Q_{T}\right)$ смешанной задачи для волнового уравнения (1) с начальными условиями $(2)$ из классов $(4)$ и с граничными условиями $u_{x}(0, t)=\mu(t), u(l, t)=\nu(t)$, в которых $\mu(t) \in L_{2}[0, T], \nu(t) \in$ $W_{2}^{1}[0, T]$ и вьполнено условие согласования $\varphi(0)=\nu(0)$, определяется как функция $u(x, t)$ из класса $\widehat{W}_{2}^{1}\left(Q_{T}\right)$, удовлетворяюшая интегральному тождеству

$$
\begin{aligned}
\int_{0}^{l} & \int_{0}^{T} u(x, t)\left[\Phi_{t t}(x, t)-\Phi_{x x}(x, t)\right] d x d t+\int_{0}^{l} \varphi(x) \Phi_{t}(x, 0) d x \\
& -\int_{0}^{l} \psi(x) \Phi(x, 0) d x+\int_{0}^{T} \mu(t) \Phi(0, t) d t+\int_{0}^{T} \nu(t) \Phi_{x}(l, t) d t=0
\end{aligned}
$$

для любой функции $\Phi(x, t)$ из класса $C^{(2)}\left(\bar{Q}_{T}\right)$, удовлетворяющей условиям $(15)$ и условиям $\Phi_{x}(0, t) \equiv 0, \Phi(l, t) \equiv 0$ при $0 \leqslant t \leqslant T$.

2) При $T>l$ функция $\widetilde{u}(x, t)$, определяемая равенством (24) с теми же $\Delta_{1}, \Delta_{2}, \Delta_{3}$ и $\Delta_{4}$, что и в задаче 2 , является решением из класса $\widehat{W}_{2}^{1}\left(Q_{T}\right)$ смешанной задачи для волнового уравнения $\widetilde{u}_{t t}(x, t)-\widetilde{u}_{x x}(x, t)=0$ с начальными условиями $\widetilde{u}(x, 0)=\varphi(x)$, $\widetilde{u}_{t}(x, 0)=\psi(x)$ и с граничными условиями

$$
\begin{aligned}
& \widetilde{u}_{x}(0, t)=\widetilde{\mu}(t)= \begin{cases}\frac{1}{2}\left[\varphi^{\prime}(t)+\psi(t)\right] & \text { при } 0 \leqslant t \leqslant l, \\
0 & \text { при } l<t \leqslant T,\end{cases} \\
& \widetilde{u}(l, t)=\widetilde{\nu}(t)=\left\{\begin{array}{l}
\frac{1}{2}\left[\varphi(l-t)+\varphi(l)+\int_{l-t}^{l} \psi(\xi) d \xi\right] \quad \text { при } 0 \leqslant t \leqslant l, \\
C_{0}=\frac{1}{2}\left[\varphi(0)+\varphi(l)+\int_{0}^{l} \psi(\xi) d \xi\right] \text { при } l \leqslant t \leqslant T .
\end{array}\right.
\end{aligned}
$$

3) При $T=4 l(n+1)$, где $n=0,1,2, \ldots$, функция $\widehat{u}(x, t)=u(x, t)-\widetilde{u}(x, t)$, являюшаяся единственньм решением из класса $\widehat{W}_{2}^{1}\left(Q_{T}\right)$ смешанной задачи для волнового уравнения $\widehat{u}_{t t}(x, t)-\widehat{u}_{x x}(x, t)=0$ с нулевыми начальными условиями $\widehat{u}(x, 0)=0$, $\widehat{u}_{t}(x, 0)=0$ и с граничными условиями $\widehat{u}_{x}(0, t)=\widehat{\mu}(t)=\mu(t)-\widetilde{\mu}(t), \widehat{u}(l, t)=\widehat{\nu}(t)=$ $\nu(t)-\widetilde{\nu}(t)$, удовлетворяюшими условию согласования $\widehat{\nu}(0)=0$, определяется равенством

$$
\begin{aligned}
\widehat{u}(x, t)= & -\sum_{k=0}^{2 n+1}(-1)^{k} \int_{0}^{t-x-2 k l} \underline{\underline{\mu}}(\tau) d \tau-\sum_{k=1}^{2 n+2}(-1)^{k} \int_{0}^{t+x-2 k l} \widehat{\underline{\mu}}(\tau) d \tau \\
& +\sum_{k=0}^{2 n+1}(-1)^{k} \underline{\widehat{\underline{\nu}}}(t-2 k l-l+x)-\sum_{k=1}^{2 n+2}(-1)^{k} \widehat{\underline{\hat{\nu}}}(t-2 k l+l-x),
\end{aligned}
$$

в котором символы $\underline{\widehat{\mu}}(t)$ и $\underline{\underline{\nu}}(t)$ обозначают функции, совпадаюшие с $\widehat{\mu}(t)$ и $\widehat{\nu}(t)$ соответственно при $0 \leqslant \bar{t} \leqslant T$ и равные нулю при $t<0$. 
4) Из равенства (46), финальных условий (3) и из условий согласования получим следуюшие три зависяшие только от разностей (13) условия связи:

$$
\left\{\begin{array}{l}
-\sum_{k=0}^{2 n+1}(-1)^{k} \mu(2 l k+x)+\sum_{k=1}^{2 n+2}(-1)^{k} \nu^{\prime}(2 l k-l+x)=\frac{1}{2}\left[\widetilde{\varphi}^{\prime}(x)+\widetilde{\psi}(x)\right], \\
\sum_{k=1}^{2 n+2}(-1)^{k} \mu(2 l k-x)+\sum_{k=0}^{2 n+1}(-1)^{k} \nu^{\prime}(2 l k+l-x)=\frac{1}{2}\left[\widetilde{\varphi}^{\prime}(x)-\widetilde{\psi}(x)\right], \\
\int_{0}^{T} \nu^{\prime}(t) d t=\widetilde{\varphi}(l),
\end{array}\right.
$$

первые два из которых являются равенствами элементов $L_{2}[0, l]$.

5) Решая задачу об отыскании минимума интеграла граничной энергии (12) с тремя условиями связи (47), мы придем к следующим выражениям для оптимальной граничной силы $u_{x}(0, t)=\mu(t)$ и оптимального граничного смешения $u(l, t)=\nu(t)$ : оптимальная граничная сила $\mu(t)$ является на сегменте $[0, T]=[0,4 l(n+1)]$ периодической функцией периода $4 l$ и для любого $k=0,1, \ldots, n$ и любого $x$ из полусегмента $0 \leqslant x<4 l$ имеет вид

$$
\mu(4 l k+x)= \begin{cases}-\frac{1}{8(n+1)}\left[\widetilde{\varphi}^{\prime}(x)+\widetilde{\psi}(x)\right] & \text { при } 0 \leqslant x<l, \\ -\frac{1}{8(n+1)}\left[\widetilde{\varphi}^{\prime}(2 l-x)-\widetilde{\psi}(2 l-x)\right] & \text { при } l \leqslant x<2 l, \\ \frac{1}{8(n+1)}\left[\widetilde{\varphi}^{\prime}(x-2 l)+\widetilde{\psi}(x-2 l)\right] & \text { при } 2 l \leqslant x<3 l, \\ \frac{1}{8(n+1)}\left[\widetilde{\varphi}^{\prime}(4 l-x)-\widetilde{\psi}(4 l-x)\right] & \text { при } 3 l \leqslant x<4 l ;\end{cases}
$$

оптимальное граничное смешение $\nu(t)$ равняется сумме двух слагаемых $\nu(t)=L(t)+$ $\alpha(t)$, первое из которых $L(t)$ является на сегменте $[0, T]$ линейной функщией вида

$$
L(t)=\widehat{\varphi}(l) \frac{t}{T}+\varphi(l)\left(1-\frac{t}{T}\right),
$$

а второе из которых $\alpha(t)$ является на сегменте $[0, T]=[0,4 l(n+1)]$ периодической функцией периода $4 l$ и для любого $k=0,1, \ldots, n$ и любого $x$ из сегмента $[0,4 l]$ имеет ВИД

$$
\alpha(4 l k+x)=\left\{\begin{aligned}
\frac{1}{8(n+1)}\left[\widetilde{\varphi}(l)-\widetilde{\varphi}(l-x)-\int_{l-x}^{l} \widetilde{\psi}(\xi) d \xi\right] & \text { при } 0 \leqslant x \leqslant l, \\
\frac{1}{8(n+1)}[\widetilde{\varphi}(l)-\widetilde{\varphi}(x-l) & \text { при } l \leqslant x \leqslant 2 l, \\
\left.-\int_{0}^{l} \widetilde{\psi}(\xi) d \xi-\int_{0}^{x-l} \widetilde{\psi}(\xi) d \xi\right] & \text { при } 2 l \leqslant x \leqslant 3 l, \\
\frac{1}{8(n+1)}[-\widetilde{\varphi}(l)+\widetilde{\varphi}(3 l-x) & \\
\frac{1}{8(n+1)}\left[-\widetilde{\varphi}(l)+\widetilde{\varphi}(x-3 l)-\int_{x-3 l}^{l} \widetilde{\psi}(\xi) d \xi\right] & \text { при } 3 l \leqslant x \leqslant 4 l .
\end{aligned}\right.
$$


6) Подчеркнем, что оптимальная граничная сила (48) зависит только от разностей $\widetilde{\varphi}(x)$ и $\widetilde{\psi}(x)$ финального и начального смещений и финальной и начальной скоростей, что интеграл от оптимальной граничной силы $\mu(t)$ по любому содержашемуся в $[0, T]$ сегменту длины $4 l$ равен нулю и что для квадрата нормы $\mu(t)$ в $L_{2}[0, T]$ справедлива оценка

$$
\int_{0}^{T} \mu^{2}(t) d t=O\left(\frac{1}{T}\right)
$$

Далее, заметим, что первый член (49) в выражении для оптимального граничного смещения $\nu(t)$ является единственной линейной функцией, обращающейся в $\varphi(l)$ при $t=0$ и в $\widehat{\varphi}(l)$ при $t=T$.

Второй член $\alpha(t)$ в выражении для $\nu(t)$ обрашается в нуль на концах сегментов периодичности (т.е. в точках $0,4 l, 8 l, \ldots, 4 l(n+1))$, равномерно на всем сегменте $[0, T]$ имеет порядок $O\left(\frac{1}{T}\right)$, а в центрах сегментов периодичности (т.е. в точках $2 l, 6 l, 10 l, \ldots$, $4 l n+2 l)$ принимает значение $-\frac{1}{4(n+1)} \int_{0}^{l} \widetilde{\psi}(\xi) d \xi$, зависящее только от разности $\widetilde{\psi}(x)=\widehat{\psi}(x)-\psi(x)$ финальной и начальной скоростей и совсем не зависяшее от начального и от финального смешений.

\section{$8^{\circ}$. Обоснование основных утверждений, используемых при проведении оптимизации в задаче 7}

Все рассмотренные выше семь задач оптимизируются по стереотипной схеме. В этом пункте мы дадим краткое обоснование всех утверждений, используемых при проведении оптимизации в задаче 7 .

Сначала докажем используемое в $2^{\circ}$ утверждение о том, что при $T>l$ функция $\widetilde{u}(x, t)$, определяемая равенством $(24)$ с $\Delta_{1}, \Delta_{2}, \Delta_{3}$ и $\Delta_{4}$, указанными в п. $2^{\circ}$, является решением из класса $\widehat{W}_{2}^{1}\left(Q_{T}\right)$ смешанной задачи для волнового уравнения $\widetilde{u}_{t t}(x, t)-$ $\widetilde{u}_{x x}(x, t)=0$ с начальными условиями $\widetilde{u}(x, 0)=\varphi(x), \widetilde{u}_{t}(x, 0)=\psi(x)$ и с граничными условиями (44) и (45).

Принадлежность этой функции классу $\widehat{W}_{2}^{1}\left(Q_{T}\right)$ очевидна, ибо эта функция в каждой из областей $\Delta_{1}, \Delta_{2}, \Delta_{3}$ и $\Delta_{4}$ представляет собой сумму функций от аргумента $(x+t)$ или $(x-t)$, имеющих суммируемую с квадратом производную, и сохраняет непрерьвность при переходе через границу любых двух из указанных четырех областей.

Достаточно доказать, что функция $\widetilde{u}(x, t)$ удовлетворяет интегральному тождеству (43), в котором $u(x, t)=\widetilde{u}(x, t), \mu(t)=\widetilde{\mu}(t), \nu(t)=\widetilde{\nu}(t)$, а $\Phi(x, t)$ - любая функция из класса $C^{(2)}\left(\bar{Q}_{T}\right)$, удовлетворяюшая условиям $(15)$ и условиям $\Phi_{x}(0, t) \equiv 0$, $\Phi(l, t) \equiv 0$ при $0 \leqslant t \leqslant T$.

Из тождества

$$
\begin{aligned}
\widetilde{u}(x, t)\left\{\Phi_{t t}(x, t)-\Phi_{x x}(x, t)\right\} \equiv\{ & {\left.\left[\widetilde{u}(x, t) \Phi_{t}(x, t)\right]_{t}-\left[\widetilde{u}(x, t) \Phi_{x}(x, t)\right]_{x}\right\} } \\
& +\left[\widetilde{u}_{x}(x, t) \Phi_{x}(x, t)-\widetilde{u}_{t}(x, t) \Phi_{t}(x, t)\right]
\end{aligned}
$$

получим, что

$$
\int_{0}^{l} \int_{0}^{T} \widetilde{u}(x, t)\left\{\Phi_{t t}(x, t)-\Phi_{x x}(x, t)\right\} d x d t=I_{1}+I_{2}
$$


где

$$
\begin{aligned}
I_{1} & =\iint_{Q_{T}}\left\{\left[\widetilde{u}(x, t) \Phi_{t}(x, t)\right]_{t}-\left[\widetilde{u}(x, t) \Phi_{x}(x, t)\right]_{x}\right\} d x d t, \\
I_{2} & =\int_{0}^{l} \int_{0}^{T}\left[\widetilde{u}_{x}(x, t) \Phi_{x}(x, t)-\widetilde{u}_{t}(x, t) \Phi_{t}(x, t)\right] d x d t .
\end{aligned}
$$

K интегралу (52) применим формулу Грина

$$
\iint_{Q_{T}}\left(\frac{\partial Q}{\partial x}-\frac{\partial P}{\partial t}\right) d x d t=\oint_{\Gamma} P d x+Q d t
$$

где $\Gamma$ - граница прямоугольника $Q_{T}$. При этом получим, что

$$
I_{1}=-\int_{\Gamma} \widetilde{u}(x, t) \Phi_{t}(x, t) d x-\int_{\Gamma} \widetilde{u}(x, t) \Phi_{x}(x, t) d t .
$$

Учитьвая, что $\Phi(x, T) \equiv \Phi_{t}(x, T) \equiv 0$ при $0 \leqslant x \leqslant l, \Phi_{x}(0, t) \equiv 0, \Phi(l, t) \equiv 0$ при $0 \leqslant t \leqslant T, \widetilde{u}(x, 0)=\varphi(x), \widetilde{u}(l, t)=\widetilde{\nu}(t)$, получим из $(54)$, что

$$
I_{1}=-\int_{0}^{l} \varphi(x) \Phi_{t}(x, 0) d x-\int_{0}^{T} \widetilde{\nu}(t) \Phi_{x}(l, t) d t .
$$

Для подсчета $I_{2}$ обозначим через $U(x, t)$ следуюшую функцию:

$$
U(x, t)=\left\{\begin{array}{lr}
\frac{1}{2}\left[\varphi(x+t)-\varphi(x-t)+\int_{0}^{x+t} \psi(\tau) d \tau+\int_{0}^{x-t} \psi(\tau) d \tau\right] & \text { в } \Delta_{1}, \\
\frac{1}{2}\left[\varphi(x+t)-\varphi(0)+\int_{0}^{x+t} \psi(\tau) d \tau\right] & \text { в } \Delta_{2}, \\
\frac{1}{2}\left[\varphi(l)-\varphi(x-t)+\int_{0}^{l} \psi(\tau) d \tau+\int_{0}^{x-t} \psi(\tau) d \tau\right] & \text { в } \Delta_{3}, \\
\frac{1}{2}\left[\varphi(l)-\varphi(0)+\int_{0}^{l} \psi(\tau) d \tau\right]=\mathrm{const} & \text { в } \Delta_{4},
\end{array}\right.
$$

и заметим, что функция $U(x, t)$ принадлежит классу $\widehat{W}_{2}^{1}\left(Q_{T}\right)$ и что почти всюду в $Q_{T}$ справедливы равенства

$$
U_{t}(x, t)=\widetilde{u}_{x}(x, t), \quad U_{x}(x, t)=\widetilde{u}_{t}(x, t) .
$$

Равенства (56) позволяют переписать (53) в виде

$$
I_{2}=\int_{0}^{l}\left[\int_{0}^{T} U_{t}(x, t) \Phi_{x}(x, t) d t\right] d x-\int_{0}^{T}\left[\int_{0}^{l} U_{x}(x, t) \Phi_{t}(x, t) d x\right] d t .
$$

Беря внутренние интегралы в (57) по частям, получим, что

$$
\begin{aligned}
I_{2}= & \int_{0}^{l}\left[U(x, T) \Phi_{x}(x, T)-U(x, 0) \Phi_{x}(x, 0)\right] d x-\int_{0}^{l} \int_{0}^{T} U(x, t) \Phi_{x t}(x, t) d x d t \\
& -\int_{0}^{T}\left[U(l, t) \Phi_{t}(l, t)-U(0, t) \Phi_{t}(0, t)\right] d t+\int_{0}^{l} \int_{0}^{T} U(x, t) \Phi_{t x}(x, t) d x d t .
\end{aligned}
$$


Учитывая взаимное уничтожение в (58) двойных интегралов, принимая во внимание равенства $\Phi_{x}(x, T) \equiv 0, U_{x}(x, 0)=\widetilde{u}_{t}(x, 0)=\psi(x), U_{t}(0, t)=\widetilde{u}_{x}(0, t)=\widetilde{\mu}(t)$, $\Phi(l, t) \equiv 0$ и используя получаемые интегрированием по частям соотношения

$$
\begin{aligned}
-\int_{0}^{l} U(x, 0) \Phi_{x}(x, 0) d x & =-U(l, 0) \Phi(l, 0)+U(0,0) \Phi(0,0)+\int_{0}^{l} U_{x}(x, 0) \Phi(x, 0) d x \\
& =U(0,0) \Phi(0,0)+\int_{0}^{l} \psi(x) \Phi(x, 0) d x \\
-\int_{0}^{T} U(l, t) \Phi_{t}(l, t) d t & =-U(l, T) \Phi(l, T)+U(l, 0) \Phi(l, 0)+\int_{0}^{T} U_{t}(l, t) \Phi(l, t) d t=0 \\
\int_{0}^{T} U(0, t) \Phi_{t}(0, t) d t & =U(0, T) \Phi(0, T)-U(0,0) \Phi(0,0)-\int_{0}^{T} U_{t}(0, t) \Phi(0, t) d t \\
& =-U(0,0) \Phi(0,0)-\int_{0}^{T} \widetilde{\mu}(t) \Phi(0, t) d t
\end{aligned}
$$

мы получим, что

$$
I_{2}=\int_{0}^{l} \psi(x) \Phi(x, 0) d x-\int_{0}^{T} \widetilde{\mu}(t) \Phi(0, t) d t .
$$

Из равенств (51), (55) и (59) вытекает справедливость доказываемого нами тождества.

Докажем теперь сформулированное в 3$)$ утверждение о том, что при $T=4 l(n+1)$, где $n=0,1,2, \ldots$, функция $\widehat{u}(x, t)$, являюшаяся решением из класса $\widehat{W}_{2}^{1}\left(Q_{T}\right)$ смешанной задачи для волнового уравнения $\widehat{u}_{t t}(x, t)-\widehat{u}_{x x}(x, t)=0$ с нулевыми начальными условиями $\widehat{u}(x, 0)=0, \widehat{u}_{t}(x, 0)=0$ и с граничными условиями $\widehat{u}_{x}(0, t)=\widehat{\mu}(t)$, $\widehat{u}(l, t)=\widehat{\nu}(t)$, в которых $\widehat{\nu}(0)=0$, определяется равенством $(46)$.

Обозначим символом $I$ следуюший интеграл:

$$
I=\int_{0}^{l} \int_{0}^{T} \widehat{u}(x, t)\left[\Phi_{t t}(x, t)-\Phi_{x x}(x, t)\right] d x d t .
$$

Переписьвая $I$ в виде

$$
I=\int_{0}^{l}\left[\int_{0}^{T} \widehat{u}(x, t) \Phi_{t t}(x, t) d t\right] d x-\int_{0}^{T}\left[\int_{0}^{l} \widehat{u}(x, t) \Phi_{x x}(x, t) d x\right] d t
$$

и производя интегрирование по частям внутренних (заключенных в квадратные скобки) интегралов, получим, что

$$
\begin{aligned}
I= & \int_{0}^{l}\left[\widehat{u}(x, T) \Phi_{t}(x, T)-\widehat{u}(x, 0) \Phi_{t}(x, 0)\right] d x-\int_{0}^{l} \int_{0}^{T} \widehat{u}_{t}(x, t) \Phi_{t}(x, t) d x d t \\
& -\int_{0}^{T}\left[\widehat{u}(l, t) \Phi_{x}(l, t)-\widehat{u}(0, t) \Phi_{x}(0, t)\right] d t+\int_{0}^{l} \int_{0}^{T} \widehat{u}_{x}(x, t) \Phi_{x}(x, t) d x d t .
\end{aligned}
$$

Учитывая, что $\Phi_{t}(x, T) \equiv 0, \widehat{u}(x, 0)=0$ при $0 \leqslant x \leqslant l, \widehat{u}(l, t)=\widehat{\nu}(t), \Phi_{x}(0, t) \equiv 0$ при $0 \leqslant t \leqslant T$, мы получим из $(61)$, что

$$
\begin{aligned}
I= & \int_{0}^{l} \int_{0}^{T} \widehat{u}_{x}(x, t) \Phi_{x}(x, t) d x d t \\
& -\int_{0}^{l} \int_{0}^{T} \widehat{u}_{t}(x, t) \Phi_{t}(x, t) d x d t-\int_{0}^{T} \widehat{\nu}(t) \Phi_{x}(l, t) d t .
\end{aligned}
$$


Введем теперь в рассмотрение функцию $U(x, t)$, положив ее на этот раз равной

$$
\begin{aligned}
U(x, t)= & \sum_{k=0}^{2 n+1}(-1)^{k} \int_{0}^{t-x-2 k l} \underline{\widehat{\mu}}(\tau) d \tau-\sum_{k=1}^{2 n+2}(-1)^{k} \int_{0}^{t+x-2 k l} \widehat{\underline{\mu}}(\tau) d \tau \\
& +\sum_{k=0}^{2 n+1}(-1)^{k} \underline{\widehat{\nu}}(t-2 k l-l+x)+\sum_{k=1}^{2 n+2}(-1)^{k} \underline{\underline{\nu}}(t-2 k l+l-x) .
\end{aligned}
$$

Тривиально проверяется, что производные функции $(63)$ и функции $\widehat{u}(x, t)$, определяемой равенством (46), почти всюду в $Q_{T}$ связаны соотношениями

$$
\widehat{u}_{x}(x, t)=U_{t}(x, t), \quad \widehat{u}_{t}(x, t)=U_{x}(x, t) .
$$

Соотношения (64) позволяют нам переписать равенство (62) в виде

$$
\begin{aligned}
I= & \int_{0}^{l}\left[\int_{0}^{T} U_{t}(x, t) \Phi_{x}(x, t) d t\right] d x \\
& -\int_{0}^{T}\left[\int_{0}^{l} U_{x}(x, t) \Phi_{t}(x, t) d x\right] d t-\int_{0}^{T} \widehat{\nu}(t) \Phi_{x}(l, t) d t .
\end{aligned}
$$

Подвергая стоящие в квадратных скобках внутренние интегралы в (65) интегрированию по частям, получим, что

$$
\begin{aligned}
I= & \int_{0}^{l}\left[U(x, T) \Phi_{x}(x, T)-U(x, 0) \Phi_{x}(x, 0)\right] d x-\int_{0}^{l} \int_{0}^{T} U(x, t) \Phi_{x t}(x, t) d x d t \\
& -\int_{0}^{T}\left[U(l, t) \Phi_{t}(l, t)-U(0, t) \Phi_{t}(0, t)\right] d t+\int_{0}^{l} \int_{0}^{T} U(x, t) \Phi_{t x}(x, t) d x d t \\
& -\int_{0}^{T} \widehat{\nu}(t) \Phi_{x}(l, t) d t .
\end{aligned}
$$

Учитьвая взаимное уничтожение двойных интегралов в (66) и равенства $\Phi_{x}(x, T) \equiv 0$, $U(x, 0) \equiv 0$ при $0 \leqslant x \leqslant l, \Phi_{t}(l, t) \equiv 0$ при $0 \leqslant t \leqslant T$, мы придадим $(66)$ следуюший вид:

$$
I=\int_{0}^{T} U(0, t) \Phi_{t}(0, t) d t-\int_{0}^{T} \widehat{\nu}(t) \Phi_{x}(l, t) d t
$$

Интегрируя первый интеграл в (67) по частям, получим

$$
\int_{0}^{T} U(0, t) \Phi_{t}(0, t) d t=U(0, T) \Phi(0, T)-U(0,0) \Phi(0,0)-\int_{0}^{T} U_{t}(0, t) \Phi(0, t) d t .
$$

Учитьвая далее, что $\Phi(0, T)=0, U(0,0)=0$ и что $U_{t}(0, t)=\widehat{u}_{x}(0, t)=\widehat{\mu}(t)$, получим из (68), что

$$
\int_{0}^{T} U(0, t) \Phi_{t}(0, t) d t=-\int_{0}^{T} \widehat{\mu}(t) \Phi(0, t) d t
$$

С помошњю последнего равенства и (67) получим, что

$$
I=-\int_{0}^{T} \widehat{\mu}(t) \Phi(0, t) d t-\int_{0}^{T} \widehat{\nu}(t) \Phi_{x}(l, t) d t .
$$


Из последнего равенства и из (60) вытекает справедливость тождества (43), взятого при $u(x, t)=\widehat{u}(x, t), \varphi(x)=0, \psi(x)=0, \mu(t)=\widehat{\mu}(t)$ и $\nu(t)=\widehat{\nu}(t)$, что и завершает доказательство утверждения, сформулированного в 3$)$.

Перейдем теперь к установлению приведенных в 4) условий связи. В силу финальных условий (3) и в силу того, что

$$
\widetilde{u}(x, T)=C_{0}=\frac{1}{2}\left[\varphi(0)+\varphi(l)+\int_{0}^{l} \psi(\xi) d \xi\right], \quad \widetilde{u}_{t}(x, T)=0,
$$

мы получим, что

$$
\widehat{u}(x, T)=\widehat{\varphi}(x)-C_{0}, \quad \widehat{u}_{t}(x, T)=\widehat{\psi}(x) .
$$

Дифференцируя равенство (46) по $x$ и по $t$, получим

$$
\begin{aligned}
\widehat{u}_{x}(x, t)= & \sum_{k=0}^{2 n+1}(-1)^{k} \underline{\widehat{\mu}}(t-x-2 k l)-\sum_{k=1}^{2 n+2}(-1)^{k} \underline{\widehat{\mu}}(t+x-2 k l) \\
& +\sum_{k=0}^{2 n+1}(-1)^{k} \underline{\underline{\underline{\nu}}^{\prime}}(t-2 k l-l+x)+\sum_{k=1}^{2 n+2}(-1)^{k} \widehat{\underline{\underline{\nu}}}^{\prime}(t-2 k l+l-x), \\
\widehat{u}_{t}(x, t)= & -\sum_{k=0}^{2 n+1}(-1)^{k} \underline{\widehat{\mu}}(t-x-2 k l)-\sum_{k=1}^{2 n+2}(-1)^{k} \underline{\widehat{\mu}}(t+x-2 k l) \\
& +\sum_{k=0}^{2 n+1}(-1)^{k} \underline{\widehat{\underline{\nu}}}^{\prime}(t-2 k l-l+x)-\sum_{k=1}^{2 n+2}(-1)^{k} \underline{\widehat{\underline{\nu}}}^{\prime}(t-2 k l+l-x) .
\end{aligned}
$$

Полагая в (70) и (71) $t=T=4 l(n+1)$, беря полусумму и полуразность получаюшихся при этом равенств и используя соотношения (69), получим два условия связи ${ }^{4}$

$$
\begin{aligned}
& -\sum_{k=1}^{2 n+2}(-1)^{k} \widehat{\mu}[2 l(2 n+2-k)+x] \\
& \quad+\sum_{k=0}^{2 n+1}(-1)^{k} \widehat{\nu}^{\prime}[2 l(2 n+2-k)-l+x]=\frac{1}{2}\left[\widehat{\varphi}^{\prime}(x)+\widehat{\psi}(x)\right], \\
& \sum_{k=0}^{2 n+1}(-1)^{k} \widehat{\mu}[2 l(2 n+2-k)-x] \\
& \quad+\sum_{k=1}^{2 n+2}(-1)^{k} \widehat{\nu}^{\prime}[2 l(2 n+2-k)+l-x]=\frac{1}{2}\left[\widehat{\varphi}^{\prime}(x)-\widehat{\psi}(x)\right],
\end{aligned}
$$

являюшиеся равенствами элементов $L_{2}[0, l]$.

\footnotetext{
${ }^{4} \mathrm{~B}(72)$ и (73) мы опустим черту под $\widehat{\mu}$ и $\widehat{\nu}^{\prime}$, ибо все стоящие в квадратных скобках аргументы этих функций неотрицательны.
} 
Учитывая теперь, что $\widehat{\mu}(t)=\mu(t)-\widetilde{\mu}(t), \widehat{\nu}(t)=\nu(t)-\widetilde{\nu}(t)$ и что для $\widetilde{\mu}(t)$ и $\widetilde{\nu}(t)$ мы располагаем явными выражениями (44) и (45), мы можем переписать условия связи $(72)$ и (73) в терминах $\mu(t)$ и $\nu(t)$ :

$$
\begin{aligned}
& -\sum_{k=1}^{2 n+2}(-1)^{k} \mu[2 l(2 n+2-k)+x] \\
& \quad+\sum_{k=0}^{2 n+1}(-1)^{k} \nu^{\prime}[2 l(2 n+2-k)-l+x]=\frac{1}{2}\left[\widetilde{\varphi}^{\prime}(x)+\widetilde{\psi}(x)\right], \\
& \sum_{k=0}^{2 n+1}(-1)^{k} \mu[2 l(2 n+2-k)-x] \\
& \quad+\sum_{k=1}^{2 n+2}(-1)^{k} \nu^{\prime}[2 l(2 n+2-k)+l-x]=\frac{1}{2}\left[\widetilde{\varphi}^{\prime}(x)-\widetilde{\psi}(x)\right] .
\end{aligned}
$$

Подчеркнем, что в правых частях (74) и (75) стоят разности (13) финальных и начальных условий.

Для получения из условий связи (74) и (75) первых двух условий связи (47) достаточно во всех суммах, стояших в $(74)$ и $(75)$, заменить индекс суммирования $k$ новым индексом суммирования $k^{\prime}=2 n+2-k$.

Итак, первые два условия связи (47) установлены. Первых двух условий связи (47) еще недостаточно, ибо эти условия не изменятся при добавлении к $\widetilde{\varphi}(x)$ произвольной постоянной.

Третье условие связи (47) сразу вытекает из условий согласования $\nu(0)=\varphi(l)$, $\nu(T)=\widehat{\varphi}(l)$ и из равенства $\widetilde{\varphi}(x)=\widehat{\varphi}(x)-\varphi(x)$.

Переходим, наконец, к приведенному в 5) отысканию минимума интеграла граничной энергии (12) с тремя условиями связи (47).

Для любого $m=0,1,2, \ldots, 4 n+3$ и любого $x$ из сегмента $[0, l]$ положим $\mu_{m}(x)=$ $\mu(l m+x), \nu_{m}(x)=\nu(l m+x)$. При этом рассматриваемая задача оптимизации сведется к отысканию минимума суммы

$$
\sum_{m=0}^{4 n+3}\left\{\int_{0}^{l} \mu_{m}^{2}(x) d x+\int_{0}^{l}\left[\nu_{m}^{\prime}(x)\right]^{2} d x\right\}
$$

с тремя условиями связи

$$
\begin{gathered}
-\sum_{m=0}^{2 n+1}(-1)^{m} \mu_{2 m}(x)-\sum_{m=0}^{2 n+1}(-1)^{m} \nu_{2 m+1}^{\prime}(x)=\frac{1}{2}\left[\widetilde{\varphi}^{\prime}(x)+\widetilde{\psi}(x)\right], \\
-\sum_{m=0}^{2 n+1}(-1)^{m} \mu_{2 m+1}(x)+\sum_{m=0}^{2 n+1}(-1)^{m} \nu_{2 m}^{\prime}(x)=\frac{1}{2}\left[\widetilde{\varphi}^{\prime}(l-x)-\widetilde{\psi}(l-x)\right], \\
\sum_{m=0}^{2 n+1} \int_{0}^{l} \nu_{2 m}^{\prime}(x) d x+\sum_{m=0}^{2 n+1} \int_{0}^{l} \nu_{2 m+1}^{\prime}(x) d x=\widetilde{\varphi}(l) .
\end{gathered}
$$

Для решения этой задачи разложим каждую из шести функций $\mu_{m}(x), \nu_{m}^{\prime}(x), \widetilde{\varphi}^{\prime}(x)$, $\widetilde{\psi}(x), \widetilde{\varphi}^{\prime}(l-x)$ и $\widetilde{\psi}(l-x)$ в ряд Фурье по произвольной полной и ортонормированной 
в $L_{2}[0, l]$ системе функций $\left\{v_{j}(x)\right\}$, содержашей в качестве нулевого элемента постоянную $v_{0}(x)=\frac{1}{\sqrt{l}}$. Обозначим $j$-е коэффищиенты Фурье указанных шести функций соответственно через $\mu_{m j}, \nu_{m j}^{\prime}, \widetilde{\varphi}_{j}^{\prime}, \widetilde{\psi}_{j}, \widetilde{\varphi}_{j}^{\prime}\langle l-x\rangle$ и $\widetilde{\psi}_{j}\langle l-x\rangle$.

В силу равенства Парсеваля рассматриваемая задача сведется к отысканию минимума суммы

$$
\sum_{j}\left\{\sum_{m=0}^{4 n+3}\left[\mu_{m j}^{2}+\left(\nu_{m j}^{\prime}\right)^{2}\right]\right\}
$$

со взятыми для любого номера $j$ условиями связи

$$
\begin{gathered}
-\sum_{m=0}^{2 n+1}(-1)^{m} \mu_{(2 m) j}-\sum_{m=0}^{2 n+1}(-1)^{m} \nu_{(2 m+1) j}^{\prime}=\frac{1}{2}\left[\widetilde{\varphi}_{j}^{\prime}+\widetilde{\psi}_{j}\right], \\
-\sum_{m=0}^{2 n+1}(-1)^{m} \mu_{(2 m+1) j}+\sum_{m=0}^{2 n+1}(-1)^{m} \nu_{(2 m) j}^{\prime}=\frac{1}{2}\left[\widetilde{\varphi}_{j}^{\prime}\langle l-x\rangle+\widetilde{\psi}_{j}\langle l-x\rangle\right]
\end{gathered}
$$

и с еще одним условием связи

$$
\sum_{m=0}^{4 n+3} \nu_{m 0}^{\prime}=\frac{\widetilde{\varphi}(l)}{\sqrt{l}} .
$$

Учитьвая, что инфимум суммы (конечной или бесконечной) не меньше суммы инфимумов ее слагаемых, будем для каждого фиксированного номера $j$ искать минимум суммы

$$
\sum_{m=0}^{4 n+3}\left[\mu_{m j}^{2}+\left(\nu_{m j}^{\prime}\right)^{2}\right]
$$

с двумя взятыми для этого номера $j$ условиями связи $\left(77^{\prime}\right)$ и $\left(78^{\prime}\right)$ в случае $j \neq 0$ и с тремя условиями связи $\left(\left(77^{\prime}\right)\right.$ и $\left(78^{\prime}\right)$, взятьми при $j=0$, и с условием $\left.\left(79^{\prime}\right)\right)$ в случае $j=0$.

Эта последняя задача элементарно решается обычньм методом Лагранжа. Если мы обозначим через $A$ постоянную $A=\frac{\widetilde{\varphi}(l)}{4 l(n+1)}$, а через $\theta_{j}$ число, равное нулю при $j \neq 0$ и равное единице при $j=0$, то решение указанной задачи записывается в виде

$$
\begin{aligned}
\mu_{(2 m) j} & =-\frac{\widetilde{\varphi}_{j}^{\prime}+\widetilde{\psi}_{j}}{8(n+1)}(-1)^{m}, \\
\mu_{(2 m+1) j} & =-\frac{\widetilde{\varphi}_{j}^{\prime}\langle l-x\rangle-\widetilde{\psi}_{j}\langle l-x\rangle}{8(n+1)}(-1)^{m}, \\
\nu_{(2 m) j}^{\prime} & =\frac{\widetilde{\varphi}_{j}\langle l-x\rangle-\widetilde{\psi}_{j}\langle l-x\rangle}{8(n+1)}(-1)^{m}+\frac{A}{\sqrt{l}} \theta_{j}, \\
\nu_{(2 m+1) j}^{\prime} & =\frac{\widetilde{\varphi}_{j}^{\prime}+\widetilde{\psi}_{j}}{8(n+1)}(-1)^{m}+\frac{A}{\sqrt{l}} \theta_{j} .
\end{aligned}
$$


Из этих соотношений заключаем, что

$$
\begin{aligned}
& \left\{\begin{array}{l}
\mu_{2 m}(x)=-\frac{\widetilde{\varphi}^{\prime}(x)+\widetilde{\psi}(x)}{8(n+1)}(-1)^{m}, \\
\mu_{2 m+1}(x)=-\frac{\widetilde{\varphi}^{\prime}(l-x)-\widetilde{\psi}(l-x)}{8(n+1)}(-1)^{m},
\end{array}\right. \\
& \left\{\begin{array}{l}
\nu_{2 m}^{\prime}(x)=\frac{\widetilde{\varphi}^{\prime}(l-x)-\widetilde{\psi}(l-x)}{8(n+1)}(-1)^{m}+A, \\
\nu_{2 m+1}^{\prime}(x)=\frac{\widetilde{\varphi}^{\prime}(x)+\widetilde{\psi}(x)}{8(n+1)}(-1)^{m}+A .
\end{array}\right.
\end{aligned}
$$

Из (80) сразу вытекает выражение (48) для оптимальной граничной силы $\mu(t)$. Для получения представления для оптимального граничного смещения $\nu(t)$ в виде суммы линейной функции (49) и добавочного члена (50) достаточно воспользоваться равенствами $(81)$, условиями согласования $\nu(0)=\varphi(l), \nu(T)=\widehat{\varphi}(l)$ и учесть, что функция $\nu(t)$ является непрерьвной на всем сегменте $0 \leqslant t \leqslant T$.

Чтобы убедиться в том, что мы нашли решение поставленной задачи оптимизации, достаточно проверить, что решение $u(x, t)$ смешанной задачи для волнового уравнения (1) с начальньми условиями (2) и с граничными условиями $u_{x}(0, t)=\mu(t)$, $u(l, t)=\nu(t)$, в качестве которых взяты найденные нами функции $\mu(t)$ и $\nu(t)$, удовлетворяет условию $u(x, T)=\widehat{\varphi}(x)$ в классическом смысле и условию $u_{t}(x, T)=\widehat{\psi}(x)$ в смысле равенства элементов $L_{2}[0, l]$. Для этого проще всего убедиться в справедливости равенств (69), для чего достаточно во взятые при $t=T=4 l(n+1)$ соотношения (46) и (71) подставить $\widehat{\mu}(t)=\mu(t)-\widetilde{\mu}(t), \widehat{\nu}(t)=\nu(t)-\widetilde{\nu}(t)$, где $\mu(t)$ и $\nu(t)-$ найденные нами оптимальные граничные управления, а $\widetilde{\mu}(t)$ и $\widetilde{\nu}(t)$ - функции, определяемые равенствами (44) и (45).

\section{Заключительное замечание}

В заключение отметим, что проблемам граничного управления колебательньми процессами, описываемыми волновым уравнением, кроме указанных вьше работ [1] и [2], были посвящены работы Ж.-Л. Лионса [5], Ф. П. Васильева [6], А. Г. Бутковского [7], А. И. Егорова [8], а проблемы оптимизации граничных управлений на физическом уровне строгости рассматривались в работе Л. Д. Акуленко [9] и в кратком изложении на строго математическом уровне - в нашем цикле работ [10]-[16] в Докладах Академии наук.

По поводу определения класса $W_{2}^{1}\left(Q_{T}\right)$ и теорем вложения в нем см. монографию С. М. Никольского [17].

\section{СПИСОК ЛИТЕРАТУРЫ}

[1] В.А.Ильин. Граничное управление процессом колебаний на двух концах в терминах обобщенного решения волнового уравнения с конечной энергией // Дифференц. уравнения. 2000. T. 36. № 11. C. 1513-1528.

[2] В.А.Ильин. Граничное управление процессом колебаний на одном конце при закрепленном втором конце в терминах обобщенного решения волнового уравнения с конечной энергией // Дифференц. уравнения. 2000. Т. 36. № 12. С. 1670-1686.

[3] В. А. Ильин. О разрешимости смешанных задач для гиперболического и параболического уравнений // УМН. 1960. Т. 15. №2. С. 97-154. 
[4] В.А. Ильин, Е. И. Моисеев. Оптимальное граничное управление упругой силой на одном конце струны при свободном втором ее конце // Дифференц. уравнения. 2005. Т. 41. № 1. C. $105-115$.

[5] J.-L. Lions. Exact controllability, stabilization and perturbations for distributed systems // SIAM Rev. 1988. V. 30. № 1. P. 1-68.

[6] Ф.П. Васильев. О двойственности в линейных задачах управления и наблюдения // Дифференц. уравнения. 1995. Т. 31. №11. С. 1893-1900.

[7] А. Г. Бутковский. Теория оптимального управления системами с распределенными параметрами. М.: Наука, 1985.

[8] А.И. Егоров. Управление упругими колебаниями // Докл. АН УССР. Сер. А. 1986. № 5. C. $60-63$.

[9] Л.Д. Акуленко. Приведение упругой системы в заданное состояние посредством силового граничного воздействия // ПММ. 1981. Т. 45. № 6. С. 1095-1103.

[10] В.А. Ильин, Е.И. Моисеев. Оптимальное граничное управление смещением на одном конце при закрепленном втором конце и отвечающее ему распределение полной энергии струны // Докл. РАН. 2004. Т. 399. №6. С. 727-731.

[11] В. А. Ильин, Е. И. Моисеев. Оптимальное граничное управление смещением на двух концах и отвечающее ему распределение полной энергии струны // Докл. РАН. 2005. Т. 400. № 1. C. $16-20$.

[12] В.А.Ильин, Е. И. Моисеев. Оптимальное граничное управление смещением на одном конце при свободном втором конце и отвечающее ему распределение полной энергии струны // Докл. РАН. 2005. Т. 400. № 5. С. 587-591.

[13] В. А. Ильин. Оптимальное граничное управление упругой силой на одном конце при закрепленном втором конце и отвечающее ему распределение полной энергии струны // Докл. PAH. 2005. T. 400. №6. C. 731-735.

[14] В.А.Ильин, Е. И. Моисеев. Оптимизация граничного управления упругой силой на одном конце струны при свободном втором ее конце // Докл. РАН. 2005. Т. 402. №1. C. $20-24$.

[15] В.А.Ильин, Е. И. Моисеев. Оптимизация граничного управления упругой силой на двух концах струны // Докл. РАН. 2005. Т. 402. № 2. С. 163-169.

[16] В.А. Ильин, Е. И. Моисеев. Оптимизация комбинированного граничного управления колебаниями струны - упругой силой на одном конце и смещением на другом конце // Докл. PAH. 2005. T. 402. № 5. С. 590-596.

[17] С. М. Никольский. Приближение функций многих переменных и теоремы вложения. М.: Наука, 1969.

Московский государственньй университет им. М. В. Ломоносова; Математический институт им. В. А. Стеклова РАН
Поступила в редакцию 06.08 .2005 